\title{
Lambda-structure on Grothendieck groups of hermitian vector bundles
}

\author{
Damien ROESSLER \\ Centre de Mathmatiques de Jussieu \\ Universit Paris 7 Denis Diderot \\ Case Postale 7012 \\ 2, place Jussieu \\ F-75251 PARIS CEDEX 05 \\ E-mail: roessler@math.jussieu.fr
}

May 16, 2006

\begin{abstract}
We define a "compactification" of the representation ring of the linear group scheme over $S p e c \mathbb{Z}$, in the spirit of Arakelov geometry. We show that it is a $\lambda$-ring which is canonically isomorphic to a localized polynomial ring and that it plays a universal role with respect to natural operations on the $K_{0}$-theory of hermitian bundles defined by Gillet-Soulé. As a byproduct, we prove that the natural pre- $\lambda$-ring structure of the $K_{0}$-theory of hermitian bundles is a $\lambda$-ring structure. This last result plays a key role in the proof of the main results of [18] and [12].
\end{abstract}

\section{Contents}

1 Introduction 2

2 Preliminaries 3

$2.1 \quad \lambda$-structures . . . . . . . . . . . . . . . . . . 3

2.2 Arithmetic Grothendieck groups . . . . . . . . . . . . . 4

3 Universal relations $\quad 6$

$3.1 \quad G$-modules........................ 7

3.2 Hermitian G-modules . . . . . . . . . . . . . . . . 7

3.2.1 First definitions and results . . . . . . . . . . 8

3.2 Tori ......................... 12

3.2.3 Linear group schemes over $\mathbb{Q} \ldots \ldots 13$

3.2.4 Linear group schemes over $\mathbb{Z} \ldots \ldots$. . . . . . . . . 16 


\section{Introduction}

In their paper [6, 6., II], Gillet and Soulé gave a definition of arithmetic Grothendieck groups, i.e. they showed how to associate Grothendieck groups to arithmetic varieties "compactified" in the sense of Arakelov geometry. Furthermore, they gave a description of a pre- $\lambda$-structure on these groups. As is the case for the traditional Grothendieck groups of vector bundles on schemes, this structure arises from the exterior power operations on vector bundles. Once this pre- $\lambda$-structure is given, one is naturally lead to ask if it is a $\lambda$-structure, i.e. if there exist universal polynomials computing products as well as compositions of $\lambda$-operations (see subsection 2.1 for a brief introduction to $\lambda$-rings). In the traditional setting of the Grothendieck groups of vector bundles mentioned above, such polynomials exist and there are two main ways to prove their existence; the first one relies on the geometric splitting of vector bundles when lifted to their own projectivized total space (see $[9, \mathrm{VI}]$ ) and the second one relies on the existence of a universal ring for natural operations on Grothendieck groups of vector bundles (see [9, p. 393] and [19]), which is a $\lambda$-ring. This last ring is in fact the representation ring of the linear group scheme over $\mathbb{Z}$. Both methods extend to the Arakelovian setting and yield the existence of a $\lambda$-structure on arithmetic Grothendieck groups. We refer to the (unpublished) text [15] for a proof using the first method. It is the purpose of the following paper to prove the existence of a universal ring for natural operations on arithmetic Grothendieck groups, which is a $\lambda$-ring and thus give a proof of the existence of a $\lambda$-structure taking the second route. It will appear that this ring is a "compactification", in the sense of Arakelov geometry, of the representation ring of the linear group scheme over $\mathbb{Z}$. The existence of such a "compactification" was apparently already known to J.-F. Burnol (private conversations) but we don't know if his methods of proof coincide with ours. This second proof apparently also works for a much more general class of schemes (Meissner's result is only formulated for projective varieties and our result applies to all schemes $X$ such that $X \times_{\mathbb{Z}} \mathbb{Q}$ is smooth over $\mathbb{Q}$ ) and differs in method from the proof of its algebraic analog (see 3.1) in that it doesn't rely on the theory of the Brauer homomorphisms for its main step. Furthermore, one might hope that the universal ring defined here could be used to define a $\lambda$-structure on a yet to be defined higher arithmetic $\widehat{K}$ theory (see [20]) since its traditional counterpart has found such an application in the paper [13]. The hermitian representations that we use to construct our "compactifications" also appear in [5], in the context of the theory of heights. The main results of this paper are announced in [16]. The existence of a special $\lambda$-structure on arithmetic Grothendieck groups plays a crucial role in the proof of the main results of [18] and [12]. (see the end of the paper). 
Acknowledgements. We want to thank C. Soulé for proposing to us the topic of these investigations and for his constant support, help and advice during our work. We also extend our thanks to J.-B. Bost and K. Künnemann, for their detailed comments on this paper.

\section{Preliminaries}

\section{$2.1 \quad \lambda$-structures}

Here we gather some general facts about $\lambda$-rings. The reader will find [1] a good reference. A pre- $\lambda$-ring is a unital commutative ring $R$ with operations $\lambda^{k}: R \rightarrow R(k \geq 0)$, such that

(i) $\lambda^{0}=1, \lambda^{1}=I d$;

(ii) $\lambda^{k}(x+y)=\sum_{i=0}^{k} \lambda^{i}(x) \lambda^{k-i}(y)$

for all $k \geq 1$ and for all $x, y \in R$. Elements $x \in R$ are called line elements if $\lambda^{i}(x)=0$ for all $i>1$. Now let $k, j \geq 0$ and $x \in R$ and suppose that $x=l_{1}+\ldots l_{r}$, where $l_{1}, \ldots l_{r}$ are line elements and that $r>k j$. The expression $\lambda^{k}\left(\lambda^{j}(x)\right)$ can then be computed via the rule (ii) and yields a polynomial with integer coefficients in the $l_{i}$, which is symmetric by construction. By the fundamental theorem on symmetric functions, this polynomial can be written as a polynomial in the symmetric functions $\sigma_{1}\left(l_{1}, \ldots l_{r}\right), \ldots \sigma_{k j}\left(l_{1}, \ldots l_{r}\right)$. which turns out to be independent of $r$; we denote it by $P_{k, l}$. Similarly, let $k \geq 0$ and let $x \in R$ (resp. $x^{\prime} \in R$ ) and suppose that $x=l_{1}+\ldots l_{r}$ (resp. $\left.x^{\prime}=l_{1}^{\prime}+\ldots l_{r^{\prime}}^{\prime}\right)$, where $l_{1}, \ldots l_{r}\left(\right.$ resp. $\left.l_{1}^{\prime}, \ldots l_{r^{\prime}}^{\prime}\right)$ are line elements and that $r>k, r^{\prime}>k$. The expression $\lambda^{k}\left(x+x^{\prime}\right)$ can then be computed via the rule (ii) and yields a polynomial with integer coefficients in the $l_{i}$ and $l_{i}^{\prime}$, which is symmetric in the $l_{i}$, as well as symmetric in the $l_{i}^{\prime}$. By the the theorem on symmetric functions it can be written as a polynomial in the symmetric functions $\sigma_{1}\left(l_{1}, \ldots, l_{r}\right), \ldots, \sigma_{k}\left(l_{1}, \ldots, l_{r}\right), \sigma_{1}\left(l_{1}^{\prime}, \ldots, l_{r^{\prime}}^{\prime}\right), \ldots, \sigma_{k}\left(l_{1}^{\prime}, \ldots, l_{r^{\prime}}^{\prime}\right)$. Again it can be proved that his polynomial is independent of $r$; we denote it by $P_{k}$.

A $\lambda$-ring is a pre- $\lambda$-ring satisfying the following additional conditions

(iii) $\lambda^{k}(x y)=P_{k}\left(\lambda^{1}(x), \ldots \lambda^{k}(x), \lambda^{1}(y), \ldots \lambda^{k}(y)\right)$;

(iv) $\lambda^{k}\left(\lambda^{l}(x)\right)=P_{k, l}\left(\lambda^{1}(x), \ldots \lambda^{k l}(x)\right)$

An element $x$ such that $\lambda^{k}(x)=0$ for all $k>d$ is said to be $\lambda$-finite of $\lambda$ dimension $d$. A pre- $\lambda$-ring with the property that all its elements are equal to differences of elements of finite $\lambda$-dimension is also said to be $\lambda$-finite. In a $\lambda$ finite ring, the conditions (iii) and (iv) are by definition automatically satisfied. 
This also holds if $R$ has a $\lambda$-ring extension in which this is satisfied.

Let $\lambda_{t}(x): R \rightarrow 1+R[[t]]$ be defined as $\lambda_{t}(x)=1+\sum_{k=1}^{\infty} \lambda^{k}(x) t^{k}$, where $1+R[[t]]$ is the multiplicative subgroup of the ring of formal power series $R[[t]]$ consisting of power series with constant coefficient 1 . The first condition states that $\lambda_{t}$ is a group homomorphism from $R$ as an additive group into $1+R[[t]]$ as a multiplicative group. A pre- $\lambda$-ring structure on $R$ gives rise to a collection of group homomorphisms, called Adams operations.

Definition 2.1 The Adams operations $\psi^{k}: R \rightarrow R$ are defined by the equations

$$
\psi_{-t}(x):=\frac{-t . d \lambda_{t}(x) / d t}{\lambda_{t}(x)}, \quad \psi_{t}(x)=: \sum_{k \geq 1} \psi^{k}(x) t^{k}
$$

The Adams operations are additive. They are multiplicative and satisfy the identities $\psi^{k} \circ \psi^{l}=\psi^{k l}(k, l \geq 1)$, if the pre- $\lambda$-structure is a $\lambda$-structure. If $R$ is a $\mathbb{Q}$-algebra and we are provided with a collection of ring endomorphisms $\psi^{k}: R \rightarrow R$, such that $\psi^{k} \circ \psi^{l}=\psi^{k l}$, we can define a $\lambda$-structure via the formula

$$
\lambda_{t}:=\exp \left(\sum_{k=1}^{\infty}(-1)^{k} \psi^{k} \cdot \frac{t^{k}}{k}\right)
$$

which inverts the formula given above for $\psi_{t}$. Given any commutative graded unitary $\mathbb{Q}$-algebra $R=\bigoplus_{i=0}^{\infty} R_{i}$, one can define Adams operations by the formula $\psi^{k}(x)=\sum_{i=0}^{\infty} k^{i} . x_{i}$, where $x_{i}$ is the component of degree $i$. Any $\mathbb{Q}$-graded ring thus carries a canonical $\lambda$-ring structure. See $[9, \mathrm{~V}$, Appendice].

\subsection{Arithmetic Grothendieck groups}

In this subsection, we recall the definition of the arithmetic Grothendieck group $\widehat{K}_{0}(X)$ of a scheme $X$ such that $X \times_{\mathbb{Z}} \mathbb{Q}$ is smooth over $\mathbb{Q}$. We describe its canonical pre- $\lambda$-ring structure.

We shall denote the complex manifold associated to its complex points by $X(\mathbb{C})$. The complex conjugation induces an anti-holomorphic automorphism $F_{\infty}$ of $X(\mathbb{C})$. A hermitian bundle $\bar{E}=(E, h)$ on $X$ is a geometric vector bundle $E$ on $X$, together with a conjugation invariant hermitian metric $h$ on $E_{\mathbb{C}}$, the holomorphic vector bundle associated to $E$ on $X(\mathbb{C})$. A metric $h$ on $E_{\mathbb{C}}$ is conjugation invariant if $\overline{\left(F_{\infty}^{*}(h)\right)(s, l)}=h(s, l)$, for all $C^{\infty}$ sections $s, l$ of $E_{\mathbb{C}}$. We shall denote by $A^{p, p}(X)$ the set of real differential forms $\omega$ of type $(p, p)$ on $X(\mathbb{C})$ that satisfy the equation $F_{\infty}^{*} \omega=(-1)^{p} \omega$ and by $\tilde{A}^{p, p}(X)$ the quotient $\left(A^{p, p}(M) /(i m \partial+i m \bar{\partial})\right)$. The direct sum $\bigoplus_{p \geq 0}\left(A^{p, p}(M) /(i m \partial+i m \bar{\partial})\right)$ will be referred to as $\tilde{A}(X)$. Finally let $Z^{p, p}(X)$ be the kernel of the operator $d=\partial+\bar{\partial}$ in $A^{p, p}(X)$ and let $Z(X)$ be the direct sum sum $\bigoplus_{p \geq 0} Z^{p, p}(X)$. Let

$$
\mathcal{E}: 0 \rightarrow E^{\prime} \rightarrow E \rightarrow E^{\prime \prime} \rightarrow 0
$$


be an exact sequence of vector bundles on $X$. Denote by $\overline{\mathcal{E}}$ the sequence $\mathcal{E}$ together with $F_{\infty}$-invariant hermitian metrics $h^{\prime}, h$ and $h^{\prime \prime}$ on $E^{\prime}, E$ and $E^{\prime \prime}$. To these data is associated a Bott-Chern secondary class $\tilde{c h}(\overline{\mathcal{E}}) \in \tilde{A}(X)$. It satisfies the equation

$$
d d^{c} \tilde{c h}(\overline{\mathcal{E}})=\operatorname{ch}\left(\bar{E}^{\prime} \oplus \bar{E}^{\prime \prime}\right)-\operatorname{ch}(\bar{E})
$$

(recall that $d^{c}=\frac{1}{4 \pi i}(\partial-\bar{\partial})$, so that $d d^{c}=\frac{1}{2 \pi i} \bar{\partial} \partial$ ) where $\operatorname{ch}\left(\bar{E}^{\prime}\right), \operatorname{ch}(\bar{E})$ and $\operatorname{ch}\left(\bar{E}^{\prime \prime}\right)$ are the Chern character forms associated to the unique holomorphic hermitian connections of $E^{\prime}, E$ and $E^{\prime \prime}$. It vanishes if the map $E^{\prime} \rightarrow E$ is metric preserving and the map $E \rightarrow E^{\prime \prime}$ admits a metric preserving holomorphic left inverse. More generally, if $\rho$ is any power series in the Chern forms associated to the holomorphic hermitian connection of a hermitian holomorphic vector bundle, one can associated a Bott-Chern secondary class $\tilde{\rho}(\overline{\mathcal{E}})$ to the same data as above, with similar properties. For a definition of the secondary classes, see [6, Th. 1.2.2, p. 167]. The following definition is taken from [6, II].

Definition 2.2 The arithmetic Grothendieck group $\widehat{K}_{0}(X)$ of $X$ is the free abelian group generated by the hermitian bundles on $X$ and $\tilde{A}(X)$, quotiented by the relations

(a) $\bar{E}+\tilde{c h}(\overline{\mathcal{E}})=\bar{E}^{\prime}+\bar{E}^{\prime \prime}$ for exact sequences of hermitian bundles $\overline{\mathcal{E}}$ as above;

(b) if $\eta^{\prime}, \eta, \eta^{\prime \prime}$ are elements of $\tilde{A}(X)$ and $\eta$ is the sum in $\tilde{A}(X)$ of $\eta^{\prime}$ and $\eta^{\prime \prime}$, then $\eta=\eta^{\prime}+\eta^{\prime \prime}$ in $\widehat{K}_{0}(X)$.

Note that there is a natural exact sequence of groups

$$
\tilde{A}(X) \stackrel{a}{\rightarrow} \widehat{K}_{0}(X) \rightarrow K_{0}(X) \rightarrow 0
$$

where the second map sends elements of $\tilde{A}(X)$ on 0 and hermitian vector bundles on the corresponding vector bundles.

One can define a multiplication and $\lambda$-operations on $\widehat{K}_{0}(X)$ as follows. Let $\Gamma(X)$ be the $\mathbb{R}$-vector space $Z(X) \oplus \tilde{A}(X)$ with the grading giving degree $p$ to the subspace $Z^{p, p} \oplus \tilde{A}^{p-1, p-1}$ when $p>0$ and degree 0 to the subspace $Z^{0,0} \oplus 0$. Denote by $*$ the bilinear pairing defined on $\Gamma(X)$ by the formula

$$
(\omega, \eta) *\left(\omega^{\prime}, \eta^{\prime}\right):=\left(\omega \wedge \omega^{\prime}, \omega \wedge \eta^{\prime}+\eta \wedge \omega^{\prime}+\left(d d^{c} \eta\right) \wedge \eta^{\prime}\right) .
$$

This pairing defines a commutative, unitary, $\mathbb{R}$-graded ring structure on $\Gamma(X)$. For a proof, see $[6,7.3 .2$, p. 233]. The space $\Gamma(X)$ is thus endowed with a canonical $\lambda$-ring structure. 
Proposition-Definition 2.3 Let $\bar{E}, \eta, \bar{E}^{\prime}, \eta^{\prime}$ be generators of $\widehat{K}_{0}(X)$. Define a product by the equations

$$
\bar{E} \cdot \bar{E}^{\prime}:=\bar{E} \otimes \bar{E}^{\prime}, \quad \eta \cdot \eta^{\prime}=\left[(0, \eta) *\left(0, \eta^{\prime}\right)\right], \quad \bar{E} \cdot \eta:=\operatorname{ch}(\bar{E}) \wedge \eta
$$

where [.] is the projection on the second component in $\Gamma(X)$. Then this product is well-defined on $\widehat{K}_{0}(X)$ and turns it into a commutative unital ring, where the unit is represented by the trivial line bundle, endowed with the trivial metric.

See $[6$, Th. 7.3 .4$, p. 235$]$ for the proof. It follows from the definitions that $\tilde{A}(X)$ is an ideal of $\Gamma(X)$ closed under the $\lambda^{k}$-operations for $k \geq 1$.

Let now $V$ be a finite dimensional complex vector space and let $h$ be a hermitian metric on $V$; let $v_{1}, \ldots v_{n}$ be a basis of $V$, which is orthonormal for $h$. The $k$ th exterior power $\lambda^{k}(V)$ of $V$ is then also endowed with a natural metric, the unique one making the standard basis $v_{i_{1}} \wedge v_{i_{2}} \wedge \ldots v_{i_{k}}\left(1 \leq i_{1}<i_{2}<\ldots i_{k} \leq n\right)$ orthonormal. One can show that the $k$-th exterior power metric is independent of the basis chosen for its construction. The basis $v_{1}, \ldots v_{n}$ also determines a representation of $s: G L_{n}(\mathbb{C}) \rightarrow \operatorname{End}(V)$ of $G L_{n}(\mathbb{C})$ in $V$; if $h^{\prime}$ is any metric on $V$, with matrix $H^{\prime}$ in the basis $v_{1}, \ldots v_{n}$, one can show from the definition that, in the standard basis consisting of the $v_{i_{1}} \wedge v_{i_{2}} \wedge \ldots v_{i_{k}}$, the matrix $H^{\prime}$ of the $k$-th exterior power metric of $h^{\prime}$ on $\lambda^{k}(V)$ is $\left(\lambda^{k}(s)\right)\left(H^{\prime}\right)$. From this, we see that the construction of the exterior power metric also carries over to vector bundles. For $k \geq 1$, let us define

$$
\lambda^{k}(\bar{E})=\lambda^{k}(E, h):=\left(\lambda^{k}(E), \lambda^{k}(h)\right)
$$

which is the $k$-th exterior power of $E$ endowed with the $k$-th exterior power metric $\lambda^{k}(h)$. We thus obtain operations $\lambda^{k}(k \geq 1)$ acting on all the generators of $\widehat{K}_{0}(X)$. The operation $\lambda^{0}$ will by convention send all the generators on the trivial line bundle. It is proved in $[6, \mathrm{II}]$ that these operations are well-defined on $\widehat{K}_{0}(X)$ and give it the structure of a pre- $\lambda$-ring.

\section{Universal relations}

In this section, we shall show that there is a "compactification" in the sense of Arakelov geometry of the representation ring of the linear group scheme over Spec $\mathbb{Z}$, the group $\widehat{R}_{\mathbb{Z}}\left(\mathbf{G L}_{n} \times \mathbf{G} \mathbf{L}_{m}\right)$, which is a $\lambda$-ring. The statement of the main result is in 3.22 , where the exact structure of that group is also given. In the last section, we shall prove that $\widehat{R}_{\mathbb{Z}}\left(\mathbf{G L}_{n} \times \mathbf{G} \mathbf{L}_{m}\right)$ plays a universal role with respect to arithmetic $K_{0}$-theory (4.4) and we shall use this universality to prove that the pre- $\lambda$-ring structure defined above on the arithmetic Grothendieck group is a $\lambda$-ring structure. 


\section{$3.1 \quad G$-modules}

In this subsection, we collect some general facts about representation theory (cf. [19]). Let $A$ be a commutative unital ring and $\mathbf{G}$ an affine group scheme over $A$. Let $E$ be an $A$-module. For any $A$-algebra $A^{\prime}$, let $\mathbf{G}\left(A^{\prime}\right)$ refer to the group of sections of $\mathbf{G}_{A^{\prime}}$ over $A^{\prime}$. This group is also called the group of points of $\mathbf{G}$ over $A^{\prime}$. Let $A u t_{A^{\prime}}(E)$ refer to the group of all $A^{\prime}$ automorphisms of $E \otimes_{A} A^{\prime}$ as an $A^{\prime}$-module. A $\mathbf{G}$-module structure on $E$ is a collection of group morphisms $d_{A^{\prime}}: \mathbf{G}\left(A^{\prime}\right) \rightarrow A u t_{A^{\prime}}(E)$, varying functorially with $A^{\prime}$. A map of G-modules is a map of $A$-modules $f: E \rightarrow E^{\prime}$ such that $\left(d_{A^{\prime}}(P)\right)\left(\left(f \otimes_{A}\right.\right.$ $I d)(v))=\left(f \otimes_{A} I d\right)\left(\left(d_{A^{\prime}}(P)\right)(v)\right)$ for all all $A$-algebras $A^{\prime}$, all $v \in E \otimes_{A} A^{\prime}$ and all $P \in \mathbf{G}\left(A^{\prime}\right)$. Furthermore, the G-modules form an abelian category. We denote the associated Grothendieck group by $R_{A}(\mathbf{G})$. If $E=A^{\oplus n}$, a $\mathbf{G}$-module structure on $E$ is equivalent to a map of group schemes from $\mathbf{G}$ to $\mathbf{G L}_{n}$, the n-dimensional linear group scheme over $A$. If $\mathbf{G}$ is the $n$-dimensional torus $\mathbf{T}^{n}$ over $A$, it can be shown (see [3, Expose I, 4.7.3]) that a $\mathbf{T}^{n}$-module structure on an $A$-module is equivalent to a $\mathbb{Z}^{n}$-grading on this module. There is a natural ring morphism $i^{*}: R_{A}\left(\mathbf{G L}_{n}\right) \rightarrow R_{A}\left(\mathbf{T}^{n}\right)$, which is the pull-back map arising from the immersion $i: \mathbf{T}^{n} \rightarrow \mathbf{G L}_{n}$. The following is a particular case of a theorem of Serre (see [19, p. 49 , Th. 4 and p. 52, 3.8]). If $R$ is a ring and $S$ a subset of $R$, we shall write $R_{S}$ for the localization of $R$ at the multiplicative set generated by $S$.

Theorem 3.1 (Serre) Let $i$ denote the natural map $\mathbf{T}^{n} \times \mathbf{T}^{m} \rightarrow \mathbf{G L}_{n} \times \mathbf{G L}_{m}$. If $A$ is a field then the induced map $i^{*}: R_{A}\left(\mathbf{G L}_{n} \times \mathbf{G} \mathbf{L}_{m}\right) \rightarrow R_{A}\left(\mathbf{T}^{n} \times \mathbf{T}^{m}\right)$ is injective and the ring $R_{A}\left(\mathbf{G L}_{n} \times \mathbf{G} \mathbf{L}_{m}\right)$ is isomorphic to the (localized) polynomial ring

$\mathbb{Z}\left[\lambda^{1}\left(I d_{n} \times 1\right), \ldots \lambda^{n}\left(I d_{n} \times 1\right), \lambda^{1}\left(1 \times I d_{m}\right), \ldots \lambda^{m}\left(1 \times I d_{m}\right)\right]_{\lambda^{n}\left(I d_{n} \times 1\right), \lambda^{m}\left(1 \times I d_{m}\right)}$. Moreover, if $A$ is a principal ideal domain with fraction field $F$ then $R_{A}\left(\mathbf{G L}_{n}\right) \simeq$ $R_{F}\left(\mathbf{G L}_{n}\right)$, where the isomorphism is induced by the functor (.) $\otimes_{A} F$.

Recall that the Weyl group of $\mathbf{T}^{n+m}$ in $\mathbf{G L}_{n} \times \mathbf{G L}_{m}$, is a finite group $W$ (see [3]), given by the quotient of the normalizer of $\mathbf{T}^{n+m}$ in $\mathbf{G} \mathbf{L}_{n} \times \mathbf{G} \mathbf{L}_{m}$ by $\mathbf{T}^{n+m}$. This group acts on $\mathbf{T}^{n+m}$ by automorphisms and is isomorphic to the symmetric group on $n+m$ elements; given a permutation $\sigma$ and a $\mathbb{Z}^{n+m}$-graded $A$-module $V=\bigoplus_{\vec{\imath} \in \mathbb{Z}^{n+m}} V_{\vec{\imath}}$, the image $\sigma^{*} V$ of $V$ under $\sigma$ is the graded module $\bigoplus_{\vec{l} \in \mathbb{Z}^{n+m}} V_{\sigma(\vec{\imath})}$, where $\sigma(\vec{\imath})$ denotes the vector $\vec{\iota}$ with components permuted by $\sigma$.

\subsection{Hermitian G-modules}

In the following, $D$ is $\mathbb{Z}$ or $\mathbb{Q}$. Let $\mathbf{G}$ be a closed group subscheme of $\left(\mathbf{G L}_{n} \times\right.$ $\left.\mathbf{G L}_{m}\right)_{D}$, with the property that the associated closed holomorphic group $\mathbf{G}(\mathbb{C}) \subseteq$ 
$\left(\mathbf{G L}_{n} \times \mathbf{G L}_{m}\right)(\mathbb{C})$ is connected for the ordinary topology and invariant under the operation of taking the matrix adjoint. This hypothesis on $\mathbf{G}$ will hold until the end of the paper. We shall construct a Grothendieck group of G-modules with metrics. This construction will turn out to yield the universal ring we seek, when applied to $\mathbf{G}=\mathbf{G L}_{n} \times \mathbf{G} \mathbf{L}_{m}$.

\subsubsection{First definitions and results}

For any finitely generated projective G-module $V$ over $D$, we let $V_{\mathbb{C}}$ be $V \otimes_{D} \mathbb{C}$ endowed with the induced $\mathbf{G}(\mathbb{C})$-module structure. Denote the corresponding group map $\mathbf{G}(\mathbb{C}) \rightarrow A u t_{\mathbb{C}}\left(V_{\mathbb{C}}\right)$ by $r$.

Definition 3.2 An admissible hermitian metric on $V$ is a conjugation invariant hermitian metric $h$ on $V_{\mathbb{C}}$ such for all $M \in \mathbf{G}(\mathbb{C}), r\left(M^{*}\right)$ is the adjoint with respect to $h$ of the endomorphism $r(M)$. The pair $\bar{V}:=(V, h)$ is called a hermitian $\mathbf{G}$-module.

Recall that the conjugation $V_{\mathbb{C}} \rightarrow V_{\mathbb{C}}$ is an $\mathbb{R}$-linear map given by the formula $v \otimes z \mapsto v \otimes \bar{z}$. A metric $h$ on $V$ is conjugation invariant if $h(\bar{x}, \bar{y})$ is the conjugate of the complex number $h(x, y)$, for all $x, y \in V_{\mathbb{C}}$.

Lemma 3.3 The representation $r$ is determined uniquely by its restriction to $\mathbf{G}(\mathbb{C}) \cap U_{n} \times U_{m}$. The metric $h$ is admissible if and only if for every $M \in$ $\mathbf{G}(\mathbb{C}) \cap U_{n} \times U_{m}$, the automorphism $r(M)$ is unitary with respect to $h$.

Here $U_{i}$ denotes the group of unitary $i \times i$ matrices.

Proof of 3.3: Recall that the underlying complex vector space of the (holomorphic) Lie algebra of $\mathbf{G L}_{n}(\mathbb{C}) \times \mathbf{G} \mathbf{L}_{m}(\mathbb{C})$ is the complex vector space $M_{n} \times M_{m}$, where $M_{i}$ is the space of complex $i \times i$ matrices. The matrix adjunction $(\cdot)^{*}$, viewed as a map from $\mathbf{G L}_{n}(\mathbb{C}) \times \mathbf{G} \mathbf{L}_{m}(\mathbb{C})$ to itself, is a $C^{\infty}$ map and its differential at the origin is the matrix adjunction viewed as a map from $M_{n} \times M_{m}$ to itself. Thus, the Lie algebra $T \mathbf{G}(\mathbb{C})$ of $\mathbf{G}(\mathbb{C})$ is invariant under matrix adjunction. Let now $M_{0} \in T \mathbf{G}(\mathbb{C})$. The elements $A:=\frac{i}{2}\left(M_{0}+M_{0}^{*}\right)$ and $B:=\frac{1}{2}\left(M_{0}-M_{0}^{*}\right)$ lie in $T \mathbf{G}(\mathbb{C})$ and by construction $M_{0}=-i . A+B$. Recall now that the (real) Lie algebra of $U_{n} \times U_{m}$ consists of the matrices $M \in M_{n} \times M_{m}$ such that $M^{*}=-M$; by construction again $A$ and $B$ lie in $T\left(U_{n} \times U_{m}\right)$ and as the representation $r$ is holomorphic we can write $r\left(M_{0}\right)=-i . r(A)+r(B)$. This settles the first statement in the lemma. To see that the second one is true suppose that for every $M \in \mathbf{G}(\mathbb{C}) \cap U_{n} \times U_{m}$, the automorphism $r(M)$ is unitary with respect to $h$ and let $M_{0}, A, B$ be as before. Consider the string of equations

$$
r\left(\exp \left(M_{0}\right)^{*}\right)=\exp \left(d r\left(M_{0}^{*}\right)\right)=\exp \left(i . d r\left(A^{*}\right)+d r\left(B^{*}\right)\right)=
$$




$$
\begin{gathered}
=\exp \left(i \cdot d r(A)^{*}+d r(B)^{*}\right)=\exp \left(d r\left(M_{0}\right)^{*}\right)=\exp \left(d r\left(M_{0}\right)\right)^{*}= \\
r\left(\exp \left(M_{0}\right)\right)^{*}
\end{gathered}
$$

These equations show that the admissibility condition is satisfied for all the elements in a small neighborhood of the identity in $T \mathbf{G}(C)$. Using the fact that matrix adjunction reverses the order of products and the fact that any neighborhood of the identity generates $T \mathbf{G}(\mathbb{C})$, we see that it is satisfied for all the elements of $T \mathbf{G}(C)$. This proves one direction of the equivalence; the other direction follows immediately from the definitions, so we are done.

Corollary 3.4 There exists an admissible metric on every finitely generated G-module.

Proof of 3.4: The group $\mathbf{G}(\mathbb{C}) \cap U_{n} \times U_{m}$ is compact and one can thus obtain a $\mathbf{G}(\mathbb{C}) \cap U_{n} \times U_{m}$-invariant metric on a representation of $\mathbf{G}(\mathbb{C})$ by integration. Using the second statement in the lemma, the result follows.

Next, we define a Grothendieck group of hermitian G-modules.

Definition 3.5 Let $\widehat{R}_{D}(\mathbf{G})$ be the free group generated by all the hermitian G-modules, with the relations $(V, h)=\left(V^{\prime}, h^{\prime}\right)+\left(V^{\prime \prime}, h^{\prime \prime}\right)$ for exact sequences

$$
0 \rightarrow V^{\prime} \rightarrow V \rightarrow V^{\prime \prime} \rightarrow 0
$$

of $\mathbf{G}$-modules such that in the induced sequence

$$
0 \rightarrow V_{\mathbb{C}}^{\prime} \rightarrow V_{\mathbb{C}} \rightarrow V_{\mathbb{C}}^{\prime \prime} \rightarrow 0
$$

the first map preserves the metric and the second map induces an isometry from the orthogonal complement of $\left(V_{\mathbb{C}}^{\prime}, h^{\prime}\right)$ in $\left(V_{\mathbb{C}}, h\right)$ onto $\left(V_{\mathbb{C}}^{\prime \prime}, h^{\prime \prime}\right)$.

A sequence as above will be said to be orthogonal.

If $\mathbf{G}$ is the trivial group scheme Spec $D$, then one might wonder if the groups $\widehat{K}_{0}(D)$ and $\widehat{R}_{D}(\mathbf{G})$ are isomorphic. The answer is affirmative; for the proof, compare [6, Th. 6.2. (i)] and [7]. We shall from now on identify $\widehat{K}_{0}(D)$ with $\widehat{R}_{D}(\mathbf{G})$ in that case.

Lemma 3.6 The orthogonal complement $\left(V_{\mathbb{C}}^{\prime}\right)^{\perp}$ of $V_{\mathbb{C}}^{\prime}$ in $V_{\mathbb{C}}$ is invariant under conjugation and is a sub-G( $\mathbb{C})$-module. In particular, in the sequence (6), the map $V_{\mathbb{C}} \rightarrow V_{\mathbb{C}}^{\prime \prime}$ has a left inverse which preserves the metric of $V_{\mathbb{C}}^{\prime \prime}$ and is a morphism of $\mathbf{G}(\mathbb{C})$-modules.

Proof of 3.6: we first prove conjugation invariance. We have to check that $\bar{x}$ is orthogonal to $V_{\mathbb{C}}$, if $x$ is orthogonal to $V_{\mathbb{C}}$. Let $y \in V_{\mathbb{C}}$; we compute $h(x, y)=$ 
$0=\overline{h(\bar{x}, \bar{y})}=h(\bar{x}, \bar{y})$. Since conjugation induces an ( $\mathbb{R}$-linear) automorphism $V_{\mathbb{C}} \rightarrow V_{\mathbb{C}}$, this implies that $h(\bar{x}, y)=0$ for all $y \in V_{\mathbb{C}}$ and thus proves our first claim. To prove the second claim, let $r: \mathbf{G}(\mathbb{C}) \rightarrow A u t_{\mathbb{C}}\left(V_{\mathbb{C}}\right)$ be the map arising from the $\mathbf{G}(\mathbb{C})$-module structure. The fact that $V_{\mathbb{C}}^{\prime}$ is a sub-G(C)-module is expressed by the relation $r(M) x \in V_{\mathbb{C}}^{\prime}$ for all $x \in V_{\mathbb{C}}^{\prime}$ and for all $M \in G(\mathbb{C})$. For $\left(V_{\mathbb{C}}\right)^{\perp}$ to be a sub-G(C)-module, the equation $h(x, r(M) y)=0$ has to hold for all $x \in V_{\mathbb{C}}^{\prime}$ and all $y \in\left(V_{\mathbb{C}}^{\prime}\right)^{\perp}$. Now using the admissibility of $h$, we can compute $h(x, r(M) y)=h\left(r\left(M^{*}\right) x, y\right)$ and $h\left(r\left(M^{*}\right) x, y\right)$ vanishes by hypothesis. Thus we are done.

Remark. The last lemma entails that the properties of $\mathbf{G}(\mathbb{C})$ that are given at the beginning of the paragraph (connectedness and invariance under the operation of taking the matrix adjoint) implie that $\mathbf{G}(\mathbb{C})$ is a reductive holomorphic group.

The two last lemmata immediately implie the following facts.

Let $V^{\prime}$ be a sub-G-module of the projective $\mathbf{G}$-module $V$. Let $h$ be an admissible metric on $V$ and $h^{\prime}$ its restriction to $V^{\prime}$. Suppose that $V / V^{\prime}$ is projective as well. When $V / V^{\prime}$ is endowed with the quotient metric, it is a hermitian $\mathbf{G}$-module . Let $(V, h),\left(V^{\prime}, h^{\prime}\right)$ be hermitian $\mathbf{G}$-modules; the tensor product metric on the $\mathbf{G}$-module $V \otimes V^{\prime}$ is admissible; the exterior power metric $\lambda^{k}(h)$ is admissible on the $\mathbf{G}$-module $\lambda^{k}(V)$.

From these facts, one deduces

Proposition 3.7 The tensor product endows the group $\widehat{R}_{D}(\mathbf{G})$ with a commutative unital ring structure.

We shall show that exterior powers are well-defined on $\widehat{R}_{D}(\mathbf{G})$.

Proposition 3.8 The equality

$$
\lambda^{r}(\bar{V})=\sum_{i=0}^{r} \lambda^{i}\left(\bar{V}^{\prime}\right) \cdot \lambda^{r-i}\left(\bar{V}^{\prime \prime}\right)
$$

holds in $\widehat{R}_{D}(\mathbf{G})$.

Proof of 3.8: let $V^{\prime}, V, V^{\prime \prime}$ be as in the Definition 3.5. The Proposition will follow from the coming lemma.

Lemma 3.9 For $i>0$, let $\lambda_{i}^{r}(V)$ be the $D$-submodule of $\lambda^{r}(V)$ generated by the elements $x_{1}^{\prime} \wedge \ldots x_{i}^{\prime} \wedge y_{i+1} \wedge \ldots \wedge y_{r}$, where $x_{1}^{\prime}, \ldots x_{i}^{\prime}$ are elements of $V^{\prime}$. For $i=0$, let $\lambda_{i}^{r}(V)$ be $\lambda^{r}(V)$. Then $\lambda_{i}^{r}(V)$ is actually a sub-G-module and there is a natural isometric isomorphism of $\mathbf{G}$-modules

$$
\lambda^{i}\left(V^{\prime}\right) \otimes \lambda^{r-i}\left(V^{\prime \prime}\right) \simeq \lambda_{i}^{r}(V) / \lambda_{i+1}^{r}(V)
$$


Proof of 3.9: for $i=0$ the statement is obvious. For $i>0$, the map is given by

$$
x_{1}^{\prime} \wedge \ldots x_{i}^{\prime} \otimes x_{1}^{\prime \prime} \wedge \ldots x_{r-i}^{\prime \prime} \rightarrow x_{1}^{\prime} \wedge \ldots x_{i}^{\prime} \wedge x_{1}^{\prime \prime} \wedge \ldots x_{r-i}^{\prime \prime}
$$

where $x_{1}^{\prime}, \ldots x_{i}^{\prime} \in V^{\prime}$ and $x_{1}^{\prime \prime}, \ldots x_{r-i}^{\prime \prime}$ are inverse images of elements of $V^{\prime \prime}$.

It is known (see [14, Prop. 9.3, p. 592]) that this map is well-defined and an isomorphism of G-modules. Let $b_{1}^{\prime}, \ldots b_{n^{\prime}}^{\prime} \in V_{\mathbb{C}}^{\prime}$ and $b_{1}^{\prime \prime}, \ldots b_{n^{\prime \prime}}^{\prime \prime} \in V_{\mathbb{C}}^{\prime \prime}$ be orthonormal bases. Considering $V_{\mathbb{C}}^{\prime \prime}$ as included in $V_{\mathbb{C}}$ by the map given in 3.6, we can consider the union $b_{1}^{\prime}, \ldots b_{n^{\prime}}^{\prime} \in V_{\mathbb{C}}^{\prime}, b_{1}^{\prime \prime}, \ldots b_{n^{\prime \prime}}^{\prime \prime} \in V_{\mathbb{C}}^{\prime \prime}$ as an orthogonal base of $V_{\mathbb{C}}$. To prove that the map (7) is an isometry amounts to showing that the elements

$$
b_{j_{1}}^{\prime} \wedge \ldots b_{j_{i}}^{\prime} \wedge b_{k_{1}}^{\prime \prime} \wedge \ldots b_{k_{r-i}}^{\prime \prime}
$$

where $1 \leq j_{1}<\ldots j_{i} \leq n^{\prime}, 1 \leq k_{1}<\ldots k_{r-i} \leq n^{\prime \prime}$, are orthonormal and in the orthogonal complement of $\lambda_{i+1}^{r}\left(V_{\mathbb{C}}\right)$. They are clearly of norm 1 . Consider now that the vector space $\lambda_{i+1}^{r}\left(V_{\mathbb{C}}\right)$ is generated by elements of the type

$$
b_{j_{1}}^{\prime} \wedge \ldots b_{j_{i+p}}^{\prime} \wedge b_{k_{1}}^{\prime \prime} \wedge \ldots b_{k_{r-i-p}}^{\prime \prime}
$$

for $p \geq 1$. These elements are all orthogonal to the elements $b_{j_{1}}^{\prime} \wedge \ldots b_{j_{i}}^{\prime} \wedge b_{k_{1}}^{\prime \prime} \wedge$ $\ldots b_{k_{r-i}}^{\prime \prime}$ mentioned above, since they are both distinct members of the canonical orthonormal basis of $\lambda^{r}\left(V_{\mathbb{C}}\right)$, arising from $b_{1}^{\prime}, \ldots b_{n^{\prime}}^{\prime}, b_{1}^{\prime \prime}, \ldots b_{n^{\prime \prime}}^{\prime \prime}$, so we are done. $\square$

Now we can compute

$$
\lambda^{r}(\bar{V})=\sum_{i=0}^{r} \lambda_{i}^{r}(V) / \lambda_{i+1}^{r}(V)=\sum_{i=0}^{r} \lambda^{i}\left(V^{\prime}\right) \otimes \lambda^{r-i}\left(V^{\prime \prime}\right)
$$

and we are done. $\square$

Theorem 3.10 The exterior powers are well-defined on $\widehat{R}_{D}(\mathbf{G})$ and endow it with the structure of a pre- $\lambda$-ring.

Proof of 3.10: let $\mathcal{I}$ be the set of all isometric isomorphism classes of hermitian G-modules. Define a map $\lambda_{t}: I \rightarrow 1+\widehat{R}_{D}(C)^{+}[[t]]$ by the formula $\lambda_{t}(\bar{V})=$ $1+\sum_{i=1}^{\infty} \lambda^{i}(\bar{V})$. Extend this map by linearity to obtain a homomorphism $\mathbb{Z}[\mathcal{I}] \rightarrow$ $1+\widehat{R}_{D}(C)^{+}[[t]]$. The Proposition 3.8 shows that the kernel of $\lambda_{t}$ contains the elements $\bar{V}-\bar{V}^{\prime}-\bar{V}^{\prime \prime}$ as above. Thus $\lambda_{t}$ and therefore all the $\lambda^{k}$ are well-defined on $\widehat{R}_{D}(\mathbf{G})$ and the fact that $\lambda_{t}$ is a homomorphism corresponds to the second part of the statement of the theorem.

There is a natural embedding of $\widehat{K}_{0}(D)$ in $\widehat{R}_{D}(\mathbf{G})$, which associates to every hermitian $D$-module the corresponding $\mathbf{G}$-module endowed with the trivial structure. We shall denote by $\widehat{\operatorname{Pic}}(D) \subseteq \widehat{K}_{0}(D)$ the isometric isomorphism classes of hermitian projective $D$-modules of rank 1 . 


\subsubsection{Tori}

In this subsubsection, we shall determine the structure of $\widehat{R}_{D}\left(\mathbf{T}^{n}\right)$. See [19] for the corresponding algebraic statements.

Lemma 3.11 A (conjugation invariant) metric $h$ on an hermitian $\mathbf{T}^{n}$-module $V$ is admissible if and only if the pieces of the $\mathbb{Z}^{n}$-grading of $\bar{V}$ are pairwise orthogonal.

Proof of 3.11: $\quad$ each $\mathbf{T}^{n}$-module structure on $V$ induces a map $S^{n}(\mathbb{C}) \stackrel{f}{\rightarrow}$ $\operatorname{Aut}_{\mathbb{C}}\left(V_{\mathbb{C}}\right)$, where $S^{n}(\mathbb{C})$ is the set of diagonal matrices in $G L_{n}(\mathbb{C})$ ). The natural grading of $V_{\mathbb{C}}$ is connected to $f$ in the following way. Let $S$ be a diagonal matrix with diagonal elements $X_{11}, X_{22}, \ldots X_{n, n}$; the piece $V_{\mathbb{C}\left(a_{1}, \ldots a_{n}\right)}$ is an eigenspace of $f(S)$ with eigenvalue $X_{11}^{a_{1}} X_{22}^{a_{2}} \ldots X_{n n}^{a_{n}}$. Now suppose that $V$ is endowed with an admissible metric. The admissibility property 3.2 implies that the images of elements of $S^{n}(\mathbb{C})$ by $f$ are normal (i.e. commute with their adjoints) for the hermitian metric of $V_{\mathbb{C}}$. For two distinct non-zero pieces of the grading $\left(a_{1}, \ldots a_{n}\right)$ and $\left(a_{1}^{\prime}, a_{2}^{\prime}, \ldots a_{n}^{\prime}\right)$ there clearly exists an element $S \in S^{n}(\mathbb{C})$, such that $V_{\mathbb{C}\left(a_{1}, \ldots a_{n}\right)}, V_{\mathbb{C}\left(a_{1}^{\prime}, a_{2}^{\prime}, \ldots a_{n}^{\prime}\right)}$ lie in distinct eigenspaces of $f(S)$. Since $f(S)$ is normal, its distinct eigenspaces are orthogonal. This implies that the pieces of the $\mathbb{Z}^{n}$-grading of $V$ are pairwise orthogonal.

To prove the converse, choose an orthonormal base on each piece of the grading and join all these bases to obtain an orthonormal base of $V_{\mathbb{C}}$, defining thus a hermitian metric on $V$. Let $S$ be a diagonal matrix as above. If we identify $f(S)$ with its matrix in this basis, $f(S)$ is diagonal and the elementson the diagonal are the polynomials $X_{11}^{a_{1}} X_{22}^{a_{2}} \ldots X_{n n}^{a_{n}}$; thus we deduce $f\left(S^{*}\right)=f(S)^{*}$. Since the adjoint of $f(S)$ for the metric $h$ can be identified with the matrix $f(S)^{*}$, this concludes the proof.

We can determine the structure of $\widehat{R}_{D}\left(\mathbf{T}^{n}\right)$ exactly. In the next Proposition, the elements $\left(e^{\vec{m}}\right)_{\vec{m} \in \mathbb{Z}^{n}}$ are the basis elements of the group algebra $\widehat{K}_{0}(D)\left[\mathbb{Z}^{n}\right]$.

Proposition 3.12 There is an isomorphism $\widehat{R}_{D}\left(\mathbf{T}^{n}\right) \stackrel{\alpha}{\simeq} \widehat{K}_{0}(D)\left[\mathbb{Z}^{n}\right]$, where $\alpha$ is defined by the formula $\alpha(\bar{V}):=\sum_{\vec{m} \in D^{n}} \bar{V}_{\vec{m}} e^{\vec{m}}$, where the $\bar{V}_{\vec{m}}$ are considered equipped with the induced metric.

Proof of 3.12: we shall describe an inverse that is a homomorphism of rings. For $\vec{m} \in \mathbb{Z}^{n}$, define a map $f_{\vec{m}}: \widehat{K}_{0}(D) \mapsto \widehat{R}_{D}\left(\mathbf{T}^{n}\right)$ which sends hermitian $D$-modules $\bar{V}$ to $\mathbf{T}^{n}$-modules with their $\vec{m}$-component equal to $\bar{V}$ and all the others 0 . This map is well-defined by definition and the family of all $f_{\vec{m}}$ defines a map $f: \widehat{K}_{0}(D)\left[\mathbb{Z}^{n}\right] \mapsto \widehat{R}_{D}\left(\mathbf{T}^{n}\right)$, which is seen to be a two-sided inverse of $\alpha$.

Proposition 3.13 All elements of $\widehat{R}_{D}\left(\mathbf{T}^{n}\right)$ are sums of line elements. 
Proof of 3.13: let $\bar{V}$ be a hermitian $\mathbf{T}^{n}$-module. Since the pieces of the grading are orthogonal by 3.11 , we can write $\bar{V}=\sum_{\vec{m} \in \mathbb{Z}^{n}} \bar{V}_{\vec{m}}$ in $\widehat{R}_{D}\left(\mathbf{T}^{n}\right)$, where the $\bar{V}_{\vec{m}}=\left(V_{\vec{m}}, h_{\vec{m}}\right)$ are considered endowed with the induced metrics and with the grading that is non-zero on the $\vec{m}$-th piece of the grading only. It will therefore be sufficient to show that any such $\bar{V}_{\vec{m}}$ can be written as sums of hermitian 1-dimensional $\mathbb{Z}^{n}$-graded modules in $\widehat{R}_{D}\left(\mathbf{T}^{n}\right)$; to prove this, remember that the structure theorem for finitely generated projective modules over a Dedekind domain (see [14, Par. 2, p. 532]) shows that there exists a fractional ideal $J$ in the quotient field of $D$ and an exact sequence of $D$-modules

$$
0 \rightarrow J \rightarrow V_{\vec{m}} \rightarrow V_{\vec{m}} / J \rightarrow 0
$$

where $V_{\vec{m}} / J$ is projective as well. Endow $J$ with the metric induced by $h$ and with the grading induced by $V_{\vec{m}}$. Endow $V_{\vec{m}} / J$ with the quotient grading and metric. One can then apply induction on the dimension to obtain the last claim and complete the proof.

\subsubsection{Linear group schemes over $\mathbb{Q}$}

In this subsubsection, we shall prove a structure theorem for $\widehat{R}_{\mathbb{Q}}\left(\mathbf{G L}_{n} \times \mathbf{G} \mathbf{L}_{m}\right)$. The proof will also show that the pre- $\lambda$-ring structure of this ring is a $\lambda$-ring structure. We let $I: R_{\mathbb{Q}}\left(\mathbf{G L}_{n} \times \mathbf{G L}_{m}\right) \simeq K_{0}(\mathbb{Q})\left[\lambda^{1}\left(I d_{n} \times 1\right), \ldots \lambda^{n}\left(I d_{n} \times\right.\right.$ 1), $\left.\lambda^{1}\left(1 \times I d_{m}\right), \ldots \lambda^{m}\left(1 \times I d_{m}\right)\right]_{\lambda^{n}\left(I d_{n} \times 1\right), \lambda^{m}\left(1 \times I d_{m}\right)}$ denote the standard isomorphism. We shall use the same letter for the standard isomorphism $I$ : $R_{\mathbb{Q}}\left(\mathbf{T}^{n+m}\right) \simeq K_{0}(\mathbb{Q})\left[\mathbb{Z}^{n+m}\right]$.

First notice that from 3.3, 3.6 and the fact that the group $U_{n} \times U_{m}$ is a maximal compact subgroup of $\left(\mathbf{G L}_{n} \times \mathbf{G L}_{m}\right)(\mathbb{C})$ we can deduce the following facts.

Let $\bar{S}$ be a simple hermitian $\left(\mathbf{G L}_{n} \times \mathbf{G} \mathbf{L}_{m}\right)_{\mathbb{Q}}$-module. Then its metric is unique up to multiplication by a constant (real, positive) factor.

Let $\bar{V}$ be a hermitian $\left(\mathbf{G} \mathbf{L}_{n} \times \mathbf{G} \mathbf{L}_{m}\right)_{\mathbb{Q}}$-module. Then there are simple $\left(\mathbf{G L}_{n} \times\right.$ $\left.\mathbf{G L}_{m}\right)_{\mathbb{Q}}$-modules with admissible metrics $\bar{V}_{1}, \ldots \bar{V}_{r}$, such that

$$
\bar{V}=\bar{V}_{1}+\ldots+\bar{V}_{r}
$$

in $\widehat{R}_{\mathbb{Q}}\left(\mathbf{G L}_{n} \times \mathbf{G L}_{m}\right)$.

Proposition 3.14 There is an exact sequence

$$
\widehat{\operatorname{Pic}}(\mathbb{Q}) \otimes_{\mathbb{Z}} R_{\mathbb{Q}}\left(\mathbf{G L}_{n} \times \mathbf{G} \mathbf{L}_{m}\right) \stackrel{\psi}{\rightarrow} \widehat{R}_{\mathbb{Q}}\left(\mathbf{G L}_{n} \times \mathbf{G L}_{m}\right) \stackrel{\phi}{\rightarrow} R_{\mathbb{Q}}\left(\mathbf{G L}_{n} \times \mathbf{G L}_{m}\right) \rightarrow 0
$$

where $\phi$ is the map forgetting the metric. The map $\psi$ is defined by

$$
\psi(a \otimes b)=(a-1) . \kappa(I(b))
$$

where $\kappa$ is the ring morphism sending $\lambda^{i}\left(I d_{n} \times 1\right)$ to $\lambda^{i}\left(\overline{I d}_{n} \times 1\right)$ and $\lambda^{i}\left(1 \times I d_{m}\right)$ to $\lambda^{i}\left(1 \times \overline{I d}_{m}\right)$. 
Proof of 3.14: we have to show that $\psi$ is well-defined. We only have to check that it is additive in the first factor, i.e. that $(a b-1)=(a-1)+$ $(b-1)$. Since the image of the inclusion $\widehat{\operatorname{Pic}}(\mathbb{Q}) \subseteq \widehat{R}_{\mathbb{Q}}\left(\mathbf{G L}_{n} \times \mathbf{G L}_{m}\right)$ lies in $\widehat{K}_{0}(\mathbb{Q}) \subseteq \widehat{R}_{\mathbb{Q}}\left(\mathbf{G L}_{n} \times \mathbf{G} \mathbf{L}_{m}\right)$, this equation has only to be checked in $\widehat{K}_{0}(\mathbb{Q})$. Now, in view of the isomorphism $K_{0}(\mathbb{Q}) \stackrel{\text { rank }}{\simeq} \mathbb{Z}$ and the sequence (4), we have $(a-1) \in \tilde{A}(\operatorname{Spec} \mathbb{Q}),(b-1) \in a(\tilde{A}(\operatorname{Spec} \mathbb{Q}))$ and by the definition of the product in $\tilde{A}$ we have $(a-1) \cdot(b-1)=0$ and thus $(a b-1)=(a-1)+(b-1)$.

Next we have to show that $\psi$ is surjective onto $\operatorname{ker} \phi$. Since $\mathbb{Q}$ is a field, the Jordan-Hoelder theorem implies that $R_{\mathbb{Q}}\left(\mathbf{G L}_{n} \times \mathbf{G} \mathbf{L}_{m}\right)$ is a free abelian group with generators the simple $\left(\mathbf{G L}_{n} \times \mathbf{G L}_{m}\right)_{\mathbb{Q}^{-m o d u l e s} \text {. Let }}$

$$
n_{1} \bar{V}_{1}+\ldots n_{r} \bar{V}_{r}
$$

be in $\operatorname{ker} \phi$. Suppose, without loss of generality, that $n_{1}, \ldots n_{r_{o}} \geq 0, n_{r_{0}+1}, \ldots n_{r} \leq$ 0 end write $\bar{V}_{i}=\bar{V}_{i 1}+\ldots \bar{V}_{i s_{i}}(1 \leq i \leq r)$ where the $\bar{V}_{i 1} \ldots$ are simple, as in 3.2.3. Under this decomposition, the expression (8) becomes

$$
n_{1} \bar{V}_{11}+n_{1} \bar{V}_{12}+\ldots-\left|n_{r_{0}+1}\right| \bar{V}_{r_{0}+1,1}-\ldots\left|n_{r}\right| \bar{V}_{r s_{r}}
$$

and the remark preceding (8) shows that the same simple modules (with multiplicities) must appear in the positive part of (9) as in its negative part. Therefore ker $\phi$ is generated by expressions $(S, h)-\left(S, h^{\prime}\right)$, where $S$ is simple. The image of $\psi$ is generated by expressions $(S, h)-(S, t . h)(t>0)$, so that we shall have proved surjectivity if we can show that the all the metrics of a simple module are proportional. This is the content of 3.2.3.

Finally, to prove surjectivity on the right, it will be sufficient to show that for every $\left(\mathbf{G L}_{n} \times \mathbf{G L}_{m}\right)_{\mathbb{C}}$-module, there exists an admissible metric. This follows from the structure theorem 3.1 for the case $A=\mathbb{C}$, the lemma 3.2.1, the Jordan-Hoelder Theorem and the lemma 3.6.

On $S p e c \mathbb{Z}$ all the line bundles are isomorphic the $\mathbb{Z}$. Thus every element of $\widehat{P i c}(\mathbb{Z})$ can be represented by a hermitian bundle of the type $(\mathbb{Z}, h)$. Moreover, it is easy to see that there is an isomorphism $\widehat{\operatorname{Pic}}(\mathbb{Z}) \simeq \mathbb{R}_{*}^{+}$sending $(\mathbb{Z}, h)$ on $\sqrt{h(1,1)}$. Similarly, there is an isomorphism $\widehat{\operatorname{Pic}}(\mathbb{Q}) \simeq \mathbb{R}_{*}^{+} / \mathbb{Q}_{*}^{+}$.

Lemma 3.15 There are isomorphisms $\widehat{K}_{0}(\mathbb{Z}) \simeq \mathbb{Z} \oplus \mathbb{R}_{*}^{+}$and $\widehat{K}_{0}(\mathbb{Q}) \simeq \mathbb{Z} \oplus$ $\mathbb{R}_{*}^{+} / \mathbb{Q}_{*}^{+}$given by $\bar{V} \mapsto \operatorname{rank}(V) \oplus \operatorname{det}(\bar{V})$.

Proof of 3.15: $\quad$ see [6, Prop. 2.5, p. 177 and Th. 7.2.1, p. 225].

Lemma 3.16 There is a commutative diagram with exact rows

$$
\begin{aligned}
& \begin{array}{cccccc}
0 & \widehat{\operatorname{Pic}}(\mathbb{Q}) \otimes_{\mathbb{Z}} R_{\mathbb{Q}}\left(\mathbf{T}^{n+m}\right) & \stackrel{\phi}{\rightarrow} & \widehat{R}_{\mathbb{Q}}\left(\mathbf{T}^{n+m}\right) & \rightarrow & R_{\mathbb{Q}}\left(\mathbf{T}^{n+m}\right) \\
\uparrow I d \otimes i^{*} & & \uparrow i^{*} & & \uparrow i^{*}
\end{array} \\
& \widehat{\operatorname{Pic}}(\mathbb{Q}) \otimes_{\mathbb{Z}} R_{\mathbb{Q}}\left(\mathbf{G L}_{n} \times \mathbf{G L}_{m}\right) \stackrel{\psi}{\rightarrow} \widehat{R}_{\mathbb{Q}}\left(\mathbf{G L}_{n} \times \mathbf{G L}_{m}\right) \quad \rightarrow \quad R_{\mathbb{Q}}\left(\mathbf{G} \mathbf{L}_{n} \times \mathbf{G L}_{m}\right) \rightarrow 0
\end{aligned}
$$


where $i$ is the natural map $\mathbf{T}^{n+m} \mapsto \mathbf{G L}_{n} \times \mathbf{G L}_{m}$ and $\phi$ is defined by

$$
\phi(a \otimes b)=(a-1) \cdot \mu(I(b))
$$

where $\mu$ sends $r . \vec{z} \in \mathbb{Z}\left[\mathbb{Z}^{n+m}\right] \simeq R_{\mathbb{Q}}\left(\mathbf{T}^{n+m}\right)$ on $\mathbb{Z}^{r}$ endowed with the standard metric and with trivial grading except at $\vec{z}$.

Proof of 3.16: only the exactness of the first row requires proof. Under the isomorphisms given in 3.12 and 3.15 , it becomes

$$
\widehat{\operatorname{Pic}}(\mathbb{Q}) \otimes_{\mathbb{Z}} \mathbb{Z}\left[\mathbb{Z}^{n+m}\right] \rightarrow(\mathbb{Z} \oplus \widehat{\operatorname{Pic}}(\mathbb{Q}))\left[\mathbb{Z}^{n+m}\right] \rightarrow \mathbb{Z}\left[\mathbb{Z}^{n+m}\right] \rightarrow 0
$$

where the first map is given by $a \otimes r \cdot \vec{z} \mapsto\left(0 \oplus a^{r}\right) . \vec{z}$ and the second one by $(r \oplus a) \cdot \vec{z} \mapsto r \cdot \vec{z}$. From this description, the surjectivity of the second map and the exactness in the middle follows. We must prove the injectivity of the first map. To prove this, notice that $\phi$ is a direct sum of morphisms $\phi_{\vec{z}}$ : $\widehat{\operatorname{Pic}}(\mathbb{Q}) \otimes_{\mathbb{Z}} \mathbb{Z} \rightarrow \widehat{\operatorname{Pic}}(\mathbb{Q})$, sending $a \otimes r$ on $a^{r}$, for all $\vec{z} \in \mathbb{Z}^{n}$. One checks that a double sided inverse to $\phi_{\vec{z}}$ is given by the map sending $a$ on $a \otimes 1$. Taking the direct sum over $\mathbb{Z}^{n}$ of these inverses yields an inverse of $\phi$ and ends the proof. $\square$

In the next corollary we shall denote by $W$ the Weyl group $W$ associated to the immersion of $\mathbf{T}^{n+m}$ in $\mathbf{G L}_{n} \times \mathbf{G L}_{m}$. If $S$ is a set on which $W$ acts, we shall write $S^{W}$ for the elements of $S$ which are fixed under each element of $W$.

Corollary 3.17 The pre- $\lambda$-structure of the ring $\widehat{R}_{\mathbb{Q}}\left(\mathbf{G L}_{n} \times \mathbf{G} \mathbf{L}_{m}\right)$ is a $\lambda$-structure. There is natural action of the Weyl group $W$ on $\widehat{R}_{\mathbb{Q}}\left(\mathbf{T}^{n+m}\right)$ and there are isomorphisms $\widehat{R}_{\mathbb{Q}}\left(\mathbf{G L}_{n} \times \mathbf{G L}_{m}\right) \stackrel{i^{*}}{\simeq}\left(\widehat{R}_{\mathbb{Q}}\left(\mathbf{T}^{n+m}\right)\right)^{W} \simeq \widehat{K}_{0}(\mathbb{Q})\left[\lambda^{1}\left(\overline{I d}_{n} \times 1\right), \ldots \lambda^{n}\left(\overline{I d}_{n} \times\right.\right.$ 1), $\left.\lambda^{1}\left(1 \times \overline{I d}_{m}\right), \ldots \lambda^{m}\left(1 \times \overline{I d}_{m}\right)\right]_{\lambda^{n}\left(\overline{I d}_{n} \times 1\right), \lambda^{m}\left(1 \times \overline{I d}_{m}\right)}$ holds.

Proof of 3.17: the commutativity of the first square in (10) and the injectivity of $\phi$ show that $\psi$ is injective too. Since the first and last vertical map are injective, we can apply the 5-lemma and conclude that the middle vertical map is injective. This proves that $\widehat{R}_{\mathbb{Q}}\left(\mathbf{G L}_{n} \times \mathbf{G L}_{m}\right)$ is a $\lambda$-ring, by the discussion before the definition of the arithmetic Grothendieck group. There is a natural action on $\widehat{\operatorname{Pic}}(\mathbb{Q}) \otimes_{\mathbb{Z}} R_{\mathbb{Q}}\left(\mathbf{T}^{n+m}\right)$ of the elements $w$ of $W$, defined by the equation $w^{*}(a \otimes$ $b)=a \otimes w^{*}(b)$. Note that an element $w$ of $W$ only modifies the $\mathbb{Z}^{n+m}$ - index of the pieces of the $\mathbb{Z}^{n+m}$-gradings of the hermitian $\mathbf{T}^{n+m}$-modules; therefore they remain pairwise orthogonal after the action of $w$, which means that $w$ sends hermitian $\mathbf{T}^{n+m}$-modules into hermitian $\mathbf{T}^{n+m}$-modules; therefore there is also a natural action of $W$ on $\widehat{R}_{\mathbb{Q}}\left(\mathbf{T}^{n+m}\right)$. Moreover, this action is compatible with the action of $W$ on $\widehat{P i c} \otimes_{\mathbb{Z}} R_{\mathbb{Q}}\left(T^{n+m}\right)$; to show this, notice that the image of $\widehat{\operatorname{Pic}}(\mathbb{Q})$ in $\widehat{R}_{\mathbb{Q}}\left(\mathbf{T}^{n+m}\right)$ is invariant under $W$, since it consists of modules with trivial grading except at 0 , whose grading is invariant under permutation of the components of the index vectors in $\mathbb{Z}^{n+m}$. We can thus compute $w^{*}(\phi(a \otimes b))=$ 
$w^{*}((a-1) \cdot \mu(I(b)))=(a-1) \cdot \mu\left(I\left(w^{*}(b)\right)\right)$, which is the compatibility. Since the isomorphisms $\widehat{\operatorname{Pic}}(\mathbb{Q}) \otimes_{\mathbb{Z}} R_{\mathbb{Q}}\left(\mathbf{G L}_{n} \times \mathbf{G} \mathbf{L}_{m}\right) \stackrel{i^{*}}{\simeq}\left(\widehat{\operatorname{Pic}}(\mathbb{Q}) \otimes_{\mathbb{Z}} R_{\mathbb{Q}}\left(\mathbf{T}^{n+m}\right)\right)^{W}$ and $R_{\mathbb{Q}}\left(\mathbf{G L}_{n} \times \mathbf{G L}_{m}\right) \stackrel{i^{*}}{\simeq}\left(R_{\mathbb{Q}}\left(\mathbf{T}^{n+m}\right)\right)^{W}$ hold, we deduce that the isomorphism $\left(\widehat{R}_{\mathbb{Q}}\left(\mathbf{T}^{n+m}\right)\right)^{W} \stackrel{i^{*}}{\simeq} \widehat{R}_{\mathbb{Q}}\left(\mathbf{G L}_{n} \times \mathbf{G L}_{m}\right)$ holds, which ends the proof.

\subsubsection{Linear group schemes over $\mathbb{Z}$}

In this subsubsection, we shall prove a structure theorem for $\widehat{R}_{\mathbb{Z}}\left(\mathbf{G L}_{n} \times \mathbf{G L}_{m}\right)$; as in the previous section the proof will show that $\widehat{R}_{\mathbb{Z}}\left(\mathbf{G L}_{n} \times \mathbf{G} \mathbf{L}_{m}\right)$ is a $\lambda$-ring. Let now $G$ satisfy the properties stated at the beginning of section 3.2 , with $D$ taken to be $\mathbb{Z}$. We denote by $R_{t}(\mathbf{G})$ the Grothendieck group of the finitely generated torsion G-modules.

Proposition 3.18 Let $E$ be $a \mathbf{G}_{\mathbb{Q}}$-module and $L_{0}$ a finitely generated $\mathbb{Z}$-module in $E$. There exists a finitely generated $\mathbf{G}$-module $L$ in $E$, containing $L_{0}$.

Proof of 3.18: see [19, p. 40, Prop. 2].

We shall define a map from $R_{t}(\mathbf{G})$ into $\widehat{R}_{\mathbb{Z}}(\mathbf{G})$ by taking projective resolutions of torsion $\mathbf{G}$-modules. For any torsion $\mathbf{G}$-module $T$, let $0 \rightarrow E \rightarrow P \stackrel{f}{\rightarrow} T \rightarrow 0$ be a resolution of $T$ by finitely generated projective $\mathbf{G - m o d u l e s . ~ S u c h ~ a ~ r e s o l u t i o n ~}$ always exists by [19, Prop. 4, p. 42]; since such a resolution determines an isomorphism $E_{\mathbb{C}} \simeq P_{\mathbb{C}}$, we can choose any admissible metric on $E_{\mathbb{C}}$ and induce it on $P_{\mathbb{C}}$, to obtain hermitian G-modules $\bar{E}$ and $\bar{P}$. We define $j(T)=\bar{P}-\bar{E}$.

Proposition 3.19 The map $j$ is a well-defined group homomorphism. The sequence

$$
R_{t}(\mathbf{G}) \stackrel{j}{\rightarrow} \widehat{R}_{\mathbb{Z}}(\mathbf{G}) \rightarrow \widehat{R}_{\mathbb{Q}}(\mathbf{G}) \rightarrow 0
$$

where the second map arises from the functor $\otimes_{\mathbb{Z}} \mathbb{Q}$, is exact.

Proof of 3.19: we shall first prove that $j$ is well-defined. Let $g$ be the morphism $E \rightarrow P$. Consider another orthogonal projective resolution $0 \rightarrow$ $E^{\prime} \stackrel{g^{\prime}}{\rightarrow} P^{\prime} \stackrel{f^{\prime}}{\rightarrow} T \rightarrow 0$. Let $Q$ be the kernel of the morphism $P \oplus P^{\prime} \stackrel{f+f^{\prime}}{\rightarrow} T$. The projection $Q \rightarrow P$ is clearly surjective and its kernel by construction arises as the image of the morphism $E^{\prime} \stackrel{0 \oplus g^{\prime}}{\rightarrow} P \oplus P^{\prime}$. Thus we get an orthogonal resolution $0 \rightarrow E^{\prime} \rightarrow Q \rightarrow P \rightarrow 0$, where $Q$ is considered endowed with the metric induced by the inclusion $Q \subseteq P \oplus P^{\prime}$. Applying precisely the same reasoning to the projection $Q \rightarrow P^{\prime}$, we get an orthogonal resolution $0 \rightarrow E \rightarrow Q \rightarrow P^{\prime} \rightarrow 0$. Thus in $\widehat{R}_{\mathbb{Z}}(\mathbf{G})$ we can compute $\bar{P}+\bar{E}^{\prime}=\bar{Q}=\bar{P}^{\prime}+\bar{E}$, which implies that $\bar{E}-\bar{P}=\bar{E}^{\prime}-\bar{P}^{\prime}$. This proves our first claim. We shall now prove that $j$ is a 
group homomorphism. Let

$$
0 \rightarrow T^{\prime} \rightarrow T \stackrel{\alpha}{\rightarrow} T^{\prime \prime} \rightarrow 0
$$

be an exact sequence of torsion $G$-modules, where $T$ is the same as before. We get another orthogonal resolution

$$
0 \rightarrow E^{\prime \prime} \rightarrow P \stackrel{f^{\prime \prime}}{\rightarrow} T^{\prime \prime} \rightarrow 0
$$

of $T^{\prime \prime}$, if we define $f^{\prime \prime}=\alpha \circ f, \bar{E}^{\prime \prime}=\overline{\operatorname{ker} f^{\prime \prime}}$, where $\overline{k e r f^{\prime \prime}}$ is endowed with the metric induced by $\bar{P}$. The snake lemma applied to the commutative diagram

$$
\begin{array}{ccccccccc}
0 & \rightarrow & E & \rightarrow & P & \stackrel{f}{\rightarrow} & T & \rightarrow 0 \\
& & \downarrow & & \downarrow & I d & & \downarrow & \\
0 & \rightarrow & E^{\prime \prime} & \rightarrow & P & \stackrel{f^{\prime \prime}}{\rightarrow} & T^{\prime \prime} & \rightarrow 0
\end{array}
$$

yields an isomorphism $T^{\prime} \simeq E^{\prime \prime} / E$ such that the resolution

$$
0 \rightarrow E \rightarrow E^{\prime \prime} \rightarrow T^{\prime} \rightarrow 0
$$

is orthogonal. Now we can compute

$$
j\left(T^{\prime}\right)-j(T)+j\left(T^{\prime \prime}\right)=\bar{E}^{\prime \prime}-\bar{E}-(\bar{P}-\bar{E})+\bar{P}-\bar{E}^{\prime \prime}=0
$$

which yields our last claim. To prove the exactness, we shall construct a map $\psi: \widehat{R}_{\mathbb{Q}}(\mathbf{G}) \rightarrow \widehat{R}_{\mathbb{Z}}(\mathbf{G}) / \operatorname{Im}(j)$ inverting the $\operatorname{map} \widehat{R}_{\mathbb{Z}}(\mathbf{G}) / \operatorname{Im}(j) \rightarrow \widehat{R}_{\mathbb{Q}}(\mathbf{G})$. Let $\bar{V}$ be a hermitian $\mathbf{G}_{\mathbb{Q}}$-module and use 3.18 to choose a lattice (i.e. a finitely generated $\mathbb{Z}$-module that generates $E$ as a $\mathbb{Q}$-vector space) $K$ in $V$ that is a G-module and induce the metric from $V$ on $K$ to obtain a hermitian G-module $\bar{K}$. Define $\psi(\bar{V})=\bar{K}$. To prove that it is well-defined, consider that if $K^{\prime}$ is another such lattice, then $K^{\prime} /\left(K^{\prime} \cap K\right)$ is a torsion $\mathbb{Z}$-module and by symmetry $K /\left(K^{\prime} \cap K\right)$ is so too. Therefore, the sequences

$$
\begin{gathered}
0 \rightarrow K^{\prime} \cap K \rightarrow K^{\prime} \rightarrow K^{\prime} /\left(K^{\prime} \cap K\right) \rightarrow 0 \\
0 \rightarrow K^{\prime} \cap K \rightarrow K \rightarrow K /\left(K^{\prime} \cap K\right) \rightarrow 0
\end{gathered}
$$

show that $\bar{K}^{\prime}=\bar{K}$ (modulo $\left.\operatorname{Im}(j)\right)$. Finally, if

$$
0 \rightarrow V^{\prime} \rightarrow V \stackrel{f}{\rightarrow} V^{\prime \prime} \rightarrow 0
$$

is an orthogonal sequence of hermitian $\mathbf{G}_{\mathbb{Q}}$-modules, we choose lattices $K^{\prime} \subseteq V^{\prime}$, $K^{\prime \prime} \subseteq V^{\prime \prime}$ as above, define $K:=f^{-1}\left(K^{\prime \prime}\right)$ and we endow them with the induced metrics. We get an orthogonal sequence

$$
0 \rightarrow K^{\prime} \rightarrow K \rightarrow K^{\prime \prime} \rightarrow 0
$$


where the maps are induced from the sequence (11), so that $\psi(\bar{V})=\psi\left(\bar{V}^{\prime}\right)+$ $\psi\left(\bar{V}^{\prime \prime}\right)$. This proves that $\psi$ is a group homomorphism and ends the whole proof.

In the next lemma, we consider the sequence of the previous Proposition, with $\mathbf{G}$ the torus of rank $n+m$ over $\mathbb{Z}$.

Lemma 3.20 The sequence of groups

$$
0 \rightarrow R_{t}\left(\mathbf{T}^{n+m}\right) \stackrel{j}{\rightarrow} \widehat{R}_{\mathbb{Z}}\left(\mathbf{T}^{n+m}\right) \rightarrow \widehat{R}_{\mathbb{Q}}\left(\mathbf{T}^{n+m}\right) \rightarrow 0
$$

is exact.

Proof of 3.20: only the injectivity of $j$ requires proof. The category of finitely generated torsion $\mathbb{Z}$-modules has a natural decomposition as a direct sum over the set of all primes $p$ of the categories of $p$-primary torsion $\mathbb{Z}$-modules. The Grothendieck group of $p$-primary torsion $\mathbb{Z}$-modules coincides with the Grothendieck group of $p$-torsion $\mathbb{Z}$-modules (modules over $\mathbb{F}_{p}$ ), because every $p$-primary $\mathbb{Z}$-module has a canonical filtration by $p$-torsion $\mathbb{Z}$-modules. Moreover, the category of finitely generated torsion $\mathbf{T}_{\mathbb{Z}}^{n+m}$-modules is canonically the direct sum over $\mathbb{Z}^{n+m}$ of the (repeated) category of finitely generated torsion $\mathbb{Z}$-modules. Using this fact and 3.12 , we see that the sequence of 3.20 can be written

$$
0 \rightarrow\left(\bigoplus_{p} K_{0}\left(\mathbb{F}_{p}\right)\right)\left[\mathbb{Z}^{n+m}\right] \stackrel{j}{\rightarrow} \widehat{K}_{0}(\mathbb{Z})\left[\mathbb{Z}^{n+m}\right] \stackrel{l}{\rightarrow} \widehat{K}_{0}(\mathbb{Q})\left[\mathbb{Z}^{n+m}\right] \rightarrow 0
$$

Since we can form projective two-term resolutions for each piece of the grading of a $\mathbf{T}^{n+m}$-module separately, this is a direct sum over $\mathbb{Z}^{n+m}$, whose elements are the unique sequence

$$
0 \rightarrow \bigoplus_{p} K_{0}\left(\mathbb{F}_{p}\right) \stackrel{j}{\rightarrow} \widehat{K}_{0}(\mathbb{Z}) \stackrel{l}{\rightarrow} \widehat{K}_{0}(\mathbb{Q}) \rightarrow 0
$$

which is the sequence (12) for $n+m=0$. Under the isomorphism of 3.15, this sequence translates to

$$
0 \rightarrow \bigoplus_{p} \mathbb{Z} \stackrel{j}{\rightarrow} \mathbb{Z} \oplus \mathbb{R}_{*}^{+} \stackrel{l}{\rightarrow} \mathbb{Z} \oplus \mathbb{R}_{*}^{+} / \mathbb{Q}_{*}^{+} \rightarrow 0
$$

The map $l$ is then the quotient map $\mathbb{Z} \oplus \mathbb{R}_{*}^{+} \rightarrow \mathbb{Z} \oplus \mathbb{R}_{*}^{+} / \mathbb{Q}_{*}^{+}$, since the functor $\otimes_{\mathbb{Z}} \mathbb{Q}$ commutes with the operation of taking the rank and taking the determinant. To determine the map $j$, we note that for any finitely generated $p$-torsion $\mathbb{Z}$-module $J$ there exist a free $\mathbb{Z}$-module $W$ so that $J \simeq W / p W$ (see [14, XV, Par. 2]). Therefore, we can write down the resolution

$$
0 \rightarrow\left(W, p^{2} . h\right) \rightarrow(W, h) \rightarrow J \rightarrow 0
$$


where the first map is multiplication by $p$ and $h$ is any metric; from this resolution we can compute

$$
\begin{gathered}
(r k \oplus \operatorname{det})(J)=(r k \oplus \operatorname{det})\left(\left(W, h \cdot p^{2}\right)-(W, h)\right)=0 \oplus \operatorname{det}(W, h) \cdot p^{r k(W)} \operatorname{det}(W, h)^{-1}= \\
0 \oplus p^{r k(W)}
\end{gathered}
$$

Therefore $j$ is given by the formula $a_{p_{1}} \oplus a_{p_{2}} \oplus \ldots \mapsto p_{1}^{a_{p_{1}}} \cdot p_{2}^{a_{p_{2}}} \ldots$ Since all the positive rational numbers can be written uniquely as products of integer powers of primes, we deduce that $j$ is injective, which completes the proof.

Proposition 3.21 The diagram

$$
\begin{aligned}
& 0 \rightarrow R_{t}\left(\mathbf{T}^{n+m}\right) \quad \rightarrow \quad \widehat{R}_{\mathbb{Z}}\left(\mathbf{T}^{n+m}\right) \quad \rightarrow \quad \widehat{R}_{\mathbb{Q}}\left(\mathbf{T}^{n+m}\right) \quad \rightarrow 0 \\
& \uparrow i^{*} \quad \uparrow i^{*} \quad \uparrow i^{*} \\
& R_{t}\left(\mathbf{G L}_{n} \times \mathbf{G L}_{m}\right) \rightarrow \widehat{R}_{\mathbb{Z}}\left(\mathbf{G L}_{n} \times \mathbf{G L}_{m}\right) \rightarrow \widehat{R}_{\mathbb{Q}}\left(\mathbf{G L}_{n} \times \mathbf{G L}_{m}\right) \rightarrow 0
\end{aligned}
$$

is commutative and the first vertical map is injective.

Proof of 3.21: the commutativity follows from the definitions. As to the second statement, notice that by construction $i^{*}$ is a direct sum over all primes $p$ of the maps $R_{\mathbb{F}_{p}}\left(\mathbf{G L}_{n} \times \mathbf{G L}_{m}\right) \stackrel{i^{*}}{\rightarrow} R_{\mathbb{F}_{p}}\left(\mathbf{T}^{n+m}\right)$, under the isomorphisms $R_{t}\left(\mathbf{T}^{n+m}\right) \simeq \bigoplus_{p} R_{\mathbb{F}_{p}}\left(\mathbf{T}^{n+m}\right)$ and $R_{t}\left(\mathbf{G L}_{n} \times \mathbf{G L}_{m}\right) \simeq \bigoplus_{p} R_{\mathbb{F}_{p}}\left(\mathbf{G L}_{n} \times \mathbf{G L}_{m}\right)$ described at the beginning of the last proof. We are therefore reduced to prove the injectivity of the maps $R_{\mathbb{F}_{p}}\left(\mathbf{G L}_{n} \times \mathbf{G L}_{m}\right) \stackrel{i^{*}}{\rightarrow} R_{\mathbb{F}_{p}}\left(\mathbf{T}^{n+m}\right)$. Since $\mathbb{F}_{p}$ is a field, this is a consequence of 3.1 .

In the next corollary, $W$ refers to Weyl group associated to the immersion $\mathbf{T}_{n} \times \mathbf{T}_{m} \rightarrow \mathbf{G L}_{n} \times \mathbf{G L}_{m}$.

Corollary 3.22 The pre- $\lambda$-structure of the ring $\widehat{R}_{\mathbb{Z}}\left(\mathbf{G L}_{n} \times \mathbf{G L}_{m}\right)$ is a $\lambda$-structure. There is a natural action of $W$ on $\widehat{R}_{\mathbb{Z}}\left(\mathbf{T}_{n} \times \mathbf{T}_{m}\right)$ and there are isomorphisms $\widehat{R}_{\mathbb{Z}}\left(\mathbf{G L}_{n} \times \mathbf{G L}_{m}\right) \simeq \widehat{R}_{\mathbb{Z}}\left(\mathbf{T}_{n} \times \mathbf{T}_{m}\right)^{W} \simeq \widehat{K}_{0}(\mathbb{Z})\left[\lambda^{1}\left(\overline{I d}_{n} \times 1\right), \ldots \lambda^{n}\left(\overline{I d}_{n} \times 1\right), \lambda^{1}(1 \times\right.$ $\left.\left.\overline{I d}_{m}\right), \ldots \lambda^{m}\left(1 \times \overline{I d}_{m}\right)\right]_{\lambda^{n}\left(\overline{I d}_{n} \times 1\right), \lambda^{m}\left(1 \times \overline{I d}_{m}\right)}$.

Proof of 3.22: consider again the diagram of 3.21. First, the injectivity of the first vertical map and of the first upper horizontal map yields the injectivity of the first lower horizontal map of the last diagram. Therefore, it follows from the 5-lemma that the middle vertical map is injective; this entails that the pre$\lambda$-structure $\widehat{R}_{\mathbb{Z}}\left(\mathbf{G L}_{n} \times \mathbf{G} \mathbf{L}_{m}\right)$ is a $\lambda$-structure. Secondly, it follows from the description of the action of the Weyl group given at the end of subsection 3.1 and the remark following (12) that the action of the Weyl group $W$ on the groups of the first row are compatible with the maps in the sequence. Now, since $W$ consists of automorphisms of $\mathbf{T}_{\mathbb{Z}}^{n+m}$, its action on the fibers of $\mathbf{T}_{\mathbb{Z}}^{n+m}$ on Spec $\mathbb{Z}$ is well-defined. It corresponds in each case to the Weyl group of $\mathbf{T}_{\mathbb{F}_{p}}^{n+m}$ in the 
fiber $\left(\mathbf{G L}_{n} \times \mathbf{G L}_{m}\right)_{\mathbb{F}_{p}}$. The correspondance is a consequence of the fact that in both cases the Weyl group is the entire symmetric group on $n+m$ elements, exchanging the components of the indices of the $\mathbb{Z}^{n+m}$ grading. Therefore the isomorphism $R_{t}\left(\mathbf{T}^{n+m}\right)^{W}=i^{*}\left(R_{t}\left(\mathbf{G L}_{n} \times \mathbf{G L}_{m}\right)\right)$ holds. From this and 3.17, we deduce that the isomorphism $\widehat{R}_{\mathbb{Z}}\left(\mathbf{G L}_{n} \times \mathbf{G L}_{m}\right) \simeq \widehat{R}_{\mathbb{Z}}\left(\mathbf{T}^{n+m}\right)^{W}$ also holds. This concludes the proof. $\square$

Open question. Is the last corollary true for a ring of integers in a number field in place of $\mathbb{Z}$ ? To prove it, one might have to extend on the left the sequence appearing in 3.19. This would probably require the definition of higher $K$ groups for the category of hermitian G-modules, since the sequence of 3.19 is a hermitian analog of the right end of Quillen's localization sequence for the torsion G-modules viewed as a (Serre) subcategory of the finitely generated G-modules.

\section{A universal action}

In this subsection, we show that $\widehat{R}_{\mathbb{Z}}\left(\mathbf{G L}_{n} \times \mathbf{G L}_{m}\right)$ is a universal object with respect to natural operations on hermitian vector bundles. This is made precise by the next theorem.

Theorem 4.1 Let $X$ be a scheme such that $X \times_{\mathbb{Z}} \mathbb{Q}$ is smooth over $\mathbb{Q}$ and $\bar{E}$ (resp. $\bar{E}^{\prime}$ ) be a hermitian vector bundle of rank $n$ (resp. $m$ ) on $X$. Then there exists a (unique) pre- $\lambda$-ring morphism $r_{\bar{E}}, \bar{E}^{\prime}: \widehat{R}_{\mathbb{Z}}\left(\mathbf{G L}_{n} \times \mathbf{G L}_{m}\right) \mapsto \widehat{K}_{0}(X)$, such that $r_{\bar{E}, \bar{E}^{\prime}}\left(\overline{I d}_{n} \times 1\right)=\bar{E}$ and $r_{\bar{E}, \bar{E}^{\prime}}\left(1 \times \overline{I d}_{m}\right)=\bar{E}^{\prime}$.

Proof of 4.1: let $h_{E}$ and $h_{E^{\prime}}$ denote the metrics of $E$ and $E^{\prime}$. The local frames of the vector bundles $E$ and $E^{\prime}$ give rise to principal bundles $\mathbf{G L}(E)$ and $\mathbf{G L}\left(E^{\prime}\right)$, called their frame bundles. By definition, $\mathbf{G L}(E)$ carries a group scheme action of $\mathbf{G L}_{n}$ and $\mathbf{G L}\left(E^{\prime}\right)$ carries a group scheme action of $\mathbf{G L}_{m}$, so that the fibre product $\mathbf{G L}(E) \times_{X} \mathbf{G L}\left(E^{\prime}\right)$ carries an action of $\mathbf{G L}_{n} \times \mathbf{G L}_{m}$. For a finitely generated projective $\mathbf{G L}_{n} \times \mathbf{G L}_{m}$-module $V$, we can form the geometric vector bundle $V\left(\mathbf{G L}(E) \times_{X} \mathbf{G L}\left(E^{\prime}\right)\right)$ associated to $V$ over $\mathbf{G L}(E) \times_{X} \mathbf{G L}\left(E^{\prime}\right)$ (cf. [9, VI, p. 396]). This construction yields an exact functor from the category of finitely generated projective $\mathbf{G L}_{n} \times \mathbf{G L}_{m}$-modules to the category of geometric vector bundles over $X$.

The metrics on the bundle $E$ and $E^{\prime}$ gives rise to $C^{\infty}$ principal bundle $U\left(E_{\mathbb{C}}\right)$ and $U\left(E_{\mathbb{C}}^{\prime}\right)$ over $X(\mathbb{C})$, the bundles of local orthonormal frames, with groups $U(n)$ and $U(m)$, the unitary groups of dimensions $n$ and $m$ (cf. [10, 7.4, p. $68])$. Endow $V$ with an admissible metric $h$ and let $f: U(n) \times U(m) \rightarrow$ $A u t_{\mathbb{C}}\left(V_{\mathbb{C}}\right)$ be the representation induced on the product of unitary groups. Write $f\left(U\left(E_{\mathbb{C}}\right) \times U\left(E_{\mathbb{C}}^{\prime}\right)\right)$ for the associated vector bundle. Again, this construction 
yields an exact functor from the category of complex linear representations of $U(n) \times U(m)$ to the category of $C^{\infty}$ vector bundles over $X(\mathbb{C})$ (cf. [10, II, 12., 5]$.$) .$

Let $N \times M \in U(n) \times U(m)$ be a product of unitary matrices. Using the admissibility of $h$ we compute $f\left((N \times M)^{*}\right)=f\left((N \times M)^{-1}\right)=(f(N \times M))^{-1}=$ $(f(N \times M))^{*}$ where $(f(N \times M))^{*}$ is the adjoint of $f(N \times M)$ for $h$. Thus $f$ sends $U(n) \times U(m)$ into the unitary subgroup of $A u t_{\mathbb{C}}\left(V_{\mathbb{C}}\right)$ determined by $h$. Let $r=\operatorname{dim}\left(V_{\mathbb{C}}\right)$ and let us choose an arbitrary orthonormal basis of $V_{\mathbb{C}}$ to identify $f$ with a morphism $f: U(n) \times U(m) \rightarrow U(r)$. We can choose an trivialising cover of $X(\mathbb{C})$ for $E_{\mathbb{C}} \times E_{\mathbb{C}}^{\prime}$ whose transition functions have image in $U(n) \times U(m)$. Now, by construction, the bundle $f\left(U\left(E_{\mathbb{C}}\right) \times U\left(E_{\mathbb{C}}^{\prime}\right)\right) \simeq$ $V\left(\mathbf{G L}(E) \times_{X} \mathbf{G L}\left(E^{\prime}\right)\right)_{\mathbb{C}}$ is (canonically isometrically isomorphic to) a bundle given by transition functions whose image lies in $U(r)$. It is thus canonically endowed with a metric, which we shall call $V\left(h_{E} \oplus h_{E}^{\prime}\right)$. Finally we define $r_{\bar{E} \bar{E}^{\prime}}(V, h):=\left(V\left(\mathbf{G L}(E) \times_{X} \mathbf{G L}\left(E^{\prime}\right)\right), V\left(h_{E} \oplus h_{E}^{\prime}\right)\right)$. The proof now follows from the next two lemmata:

Lemma 4.2 The metric $V\left(h_{E} \oplus h_{E}^{\prime}\right)$ is conjugation invariant.

Proof of 4.2: the construction of the bundle

$\left(V\left(\mathbf{G L}(E) \times_{X} \mathbf{G L}\left(E^{\prime}\right)\right), V\left(h_{E} \oplus h_{E}^{\prime}\right)\right)$ is clearly local on $X$, so we may assume that $X$ is an affine scheme where $E$ and $E^{\prime}$ are free. Since it is of finite type over $\mathbb{Z}$, we have $X \simeq \operatorname{Spec} \mathbb{Z}\left[X_{1}, X_{2}, \ldots X_{i}\right] /\left(f_{1}, \ldots f_{j}\right)$. By definition, the manifold $X(\mathbb{C})$ is then given by the zero set in $\mathbb{C}^{i}$ of the polynomials $f_{1}, \ldots f_{j}$ and the total space of $E_{\mathbb{C}} \times E_{\mathbb{C}}^{\prime}$ is then $X(\mathbb{C}) \times \mathbb{C}^{n+m}$. For every element $v \times w \in X(\mathbb{C}) \times \mathbb{C}^{n+m}$, we have $F_{\infty}^{*}(v \times w)=\bar{v} \times \bar{w}$, where the overbar $\overline{(.)}$ refers to complex conjugation of the coefficients. Let $H: X(\mathbb{C}) \rightarrow G L_{n}(\mathbb{C}) \times G L_{m}(\mathbb{C})$ be the function giving the matrix of the metric of $E_{\mathbb{C}} \times E_{\mathbb{C}}^{\prime}$ in the standard basis of $\mathbb{C}^{n+m}$. The total space of $V\left(\mathbf{G L}(E) \times_{X} \mathbf{G L}\left(E^{\prime}\right)\right)_{\mathbb{C}} \simeq V_{\mathbb{C}}\left(\mathbf{G L}\left(E_{\mathbb{C}}\right) \times_{X(\mathbb{C})} \mathbf{G L}\left(E_{\mathbb{C}}^{\prime}\right)\right)$ is then $X(\mathbb{C}) \times V_{\mathbb{C}}$ and its metric is given by the formula $h((),. r(H)()$.$) , where r$ is the associated complex representation as in 3.2. Now notice that

(a) $H(\bar{v})=\bar{H}(v)$, where $\bar{H}(v)$ is the complex conjugate of the matrix $H(v)$.

(b) $r(\bar{M})(x)=\overline{r(M)(\bar{x})}$ for all $M \in G L_{n}(\mathbb{C}) \times G L_{m}(\mathbb{C})$ and all $x \in V_{\mathbb{C}}$, where $\bar{x}$ is the conjugate of $x$ for the natural conjugation on $V_{\mathbb{C}}$.

To prove (a), note that by the conjugation invariance of the metric, we have $w_{1}^{*} H(v) w_{2}={\overline{w_{1}^{*}} H(\bar{v}) \bar{w}_{2}}^{*}=w_{1}^{*} \bar{H}(\bar{v}) w_{2}$ for all $w_{1}, w_{2}$ over $v$. To prove (b), let us choose a basis of $V$, to obtain an integral basis of $V_{\mathbb{C}}$ by base change. For the time of the next paragraph, identify $r$ with a map $G L_{n}(\mathbb{C}) \times G L_{m}(\mathbb{C}) \rightarrow$ $G L_{d}(\mathbb{C})$, where $d$ is the dimension of $V$. The matrix coefficients of $r$ are then rational functions with integer coefficients in the components of the elements of $G L_{n}(\mathbb{C}) \times G L_{m}(\mathbb{C})$. Thus we see, that $r(\bar{M})=\overline{r(M)}$, where $\overline{r(M)}$ is the 
complex conjugate of $r(M)$ in $G L_{d}(\mathbb{C})$. This proves the claim.

Using the conjugation invariance requirement for $h$, (a) and (b), we can now compute

$$
h(x, r(H(v)) y)=\overline{h(\bar{x}, \overline{r(H(v)) y})}=\overline{h(\bar{x}, r(\bar{H}(v)) \bar{y})}=\overline{h(\bar{x}, r(H(\bar{v})) \bar{y})}
$$

which proves that the metric on $V\left(\mathbf{G L}(E) \times{ }_{X} \mathbf{G} \mathbf{L}\left(E^{\prime}\right)\right)_{\mathbb{C}}$ is conjugation invariant and ends the proof. $\square$

Lemma 4.3 The map $r_{\bar{E}, \bar{E}^{\prime}}$ is well-defined as a map from $\widehat{R}_{\mathbb{Z}}\left(\mathbf{G L}_{n} \times \mathbf{G L}_{m}\right)$ and is a pre- $\lambda$-ring morphism.

Proof of 4.3: consider

$$
0 \rightarrow V^{\prime} \rightarrow V \rightarrow V^{\prime \prime} \rightarrow 0
$$

an exact sequence of $\mathbf{G L}_{n} \times \mathbf{G L}_{m}$-modules, endowed with admissible metrics $h^{\prime}, h$ and $h^{\prime \prime}$, which is orthogonal. To prove well-definedness, we have to prove that the sequence

$0 \rightarrow V^{\prime}\left(\mathbf{G L}(E) \times{ }_{X} \mathbf{G L}\left(E^{\prime}\right)\right) \rightarrow V\left(\mathbf{G L}(E) \times{ }_{X} \mathbf{G L}\left(E^{\prime}\right)\right) \rightarrow V^{\prime \prime}\left(\mathbf{G L}(E) \times_{X} \mathbf{G L}\left(E^{\prime}\right)\right) \rightarrow 0$

has a vanishing secondary class. Let $f^{\prime}, f$ and $f^{\prime \prime}$ be the representations of $U(n) \times U(m)$ induced by $V^{\prime}, V$ and $V^{\prime \prime}$. Until the end of the paragraph, consider $V_{\mathbb{C}}^{\prime}, V_{\mathbb{C}}$ and $V_{\mathbb{C}}^{\prime \prime}$ as representation spaces of $U(n) \times U(m)$. Let $\left(V_{\mathbb{C}}^{\prime}\right)^{*},\left(V_{\mathbb{C}}\right)^{*}$ and $\left(V_{\mathbb{C}}^{\prime \prime}\right)^{*}$ be the dual representations. The fact that the map $V^{\prime} \rightarrow V$ preserves the metrics is expressed by the commutativity of the diagram

$$
\begin{array}{cc}
\left(V_{\mathbb{C}}^{\prime}\right)^{*} \longleftarrow & \left(V_{\mathbb{C}}\right)^{*} \\
\uparrow & \uparrow \\
V_{\mathbb{C}}^{\prime} \longrightarrow & V_{\mathbb{C}}
\end{array}
$$

where the two vertical arrows are the anti-linear isomorphisms of representations induced by the metrics and the upper horizontal arrow is the dual of the lower horizontal one. By functoriality, this diagram yields the commutative diagram of vector bundles

$$
\begin{array}{rrr}
\left(f^{\prime}\left(U\left(E_{\mathbb{C}}\right) \times U\left(E_{\mathbb{C}}^{\prime}\right)\right)\right)^{*} \longleftarrow & \left(f\left(U\left(E_{\mathbb{C}}\right) \times U\left(E_{\mathbb{C}}^{\prime}\right)\right)\right)^{*} \\
f^{\prime}\left(U\left(E_{\mathbb{C}}\right) \times U\left(E_{\mathbb{C}}^{\prime}\right)\right) & \longrightarrow & f\left(U\left(E_{\mathbb{C}}\right) \times U\left(E_{\mathbb{C}}^{\prime}\right)\right)
\end{array}
$$

which in turn implies that the map

$$
\left(V^{\prime}\left(\mathbf{G L}(E) \times_{X} \mathbf{G L}\left(E^{\prime}\right)\right)\right)_{\mathbb{C}} \rightarrow\left(V\left(\mathbf{G L}(E) \times_{X} \mathbf{G} \mathbf{L}\left(E^{\prime}\right)\right)\right)_{\mathbb{C}}
$$

preserves the hermitian metrics. By the same token, the left inverse $V_{\mathbb{C}}^{\prime \prime} \rightarrow V_{\mathbb{C}}$ appearing in lemma 3.6, yields a metric preserving holomorphic map $\left(V^{\prime \prime}\left(\mathbf{G L}(E) \times_{X}\right.\right.$ 
$\left.\left.\mathbf{G L}\left(E^{\prime}\right)\right)\right)_{\mathbb{C}} \rightarrow\left(V\left(\mathbf{G L}(E) \times_{X} \mathbf{G L}\left(E^{\prime}\right)\right)\right)_{\mathbb{C}}$, which splits the sequence (14) orthogonally. By the remark preceding the definition of the arithmetic Grothendieck group, this shows that the secondary class of (14) vanishes. The map $r_{\bar{E}}, \bar{E}^{\prime}$ is a pre- $\lambda$-ring morphism by construction, so we are done.

Corollary 4.4 Let $X$ be a scheme such that $X \times_{\mathbb{Z}} \mathbb{Q}$ is smooth over $\mathbb{Q}$. Then $\widehat{K}_{0}(X)$ is a $\lambda$-ring.

Proof of 4.4: we want to prove (iii) and (iv) in 2.1. Assume $x=\bar{E}$ and $y=\bar{E}^{\prime}$. Then 4.1 tells us that the image of $r_{\bar{E}, \bar{E}^{\prime}}$ contains $x$ and $y$. By 3.22 all the pairs of elements of $\widehat{R}_{\mathbb{Z}}\left(\mathbf{G L}_{n} \times \mathbf{G L}_{m}\right)$ satisfy $(i i i)$ and $(i v)$. This implies that they hold for $x$ and $y$ since $r_{\bar{E}}, \bar{E}^{\prime}$ is a pre- $\lambda$-ring morphism. Now notice that if (iii) holds for $x=x^{\prime}$ and $y=y^{\prime}$ (resp. $x=x^{\prime \prime}$ and $y=y^{\prime \prime}$ ) then it holds for $x=x^{\prime}+x^{\prime}$ and $y=y^{\prime}+y^{\prime \prime}$; this follows from the construction of the polynomials $P_{k}$ and $P_{k, l}$ (see subsection 2.1) and the unicity statement in the fundamental theorem on symmetric functions; similarly, if (iv) holds for $x=x^{\prime}$ (resp. $x=x^{\prime \prime}$ ) then it holds for $x=x^{\prime}+x^{\prime \prime}$. We are thus reduced to the cases (a) $x=(0, \eta), y=\left(0, \eta^{\prime}\right)$, where $\eta, \eta^{\prime} \in \tilde{A}(X)$ and (b) $x=(0, \eta), y=\bar{E}$, where $\eta \in \tilde{A}(X)$. The case (a) is settled by the remark following 2.3. To prove (b), we compute

$$
\begin{gathered}
\lambda^{k}(\bar{E} \cdot \eta)=\lambda^{k}((0,[(\operatorname{ch}(\bar{E}), 0) *(0, \eta)]))= \\
\left(0,\left[\lambda^{k}((\operatorname{ch}(\bar{E}), 0) *(0, \eta))\right]\right)= \\
\left(0,\left[P_{k}\left(\lambda^{1}((\operatorname{ch}(\bar{E}), 0)), \ldots \lambda^{k}((\operatorname{ch}(\bar{E}), 0)), \lambda^{1}((0, \eta)), \ldots \lambda^{k}((0, \eta))\right)\right]\right)= \\
\left(0,\left[P_{k}\left(\left(\operatorname{ch}\left(\lambda^{1}(\bar{E})\right), 0\right), \ldots\left(\operatorname{ch}\left(\lambda^{k}(\bar{E})\right), 0\right), \lambda^{1}((0, \eta)), \ldots \lambda^{k}((0, \eta))\right)\right]\right)= \\
P_{k}\left(\lambda^{1}(\bar{E}), \ldots \lambda^{k}(\bar{E}), \ldots \lambda^{1}(\eta), \ldots \lambda^{k}(\eta)\right)
\end{gathered}
$$

where we used the equality $\lambda^{k}((\operatorname{ch}(\bar{E}), 0))=\left(\operatorname{ch}\left(\lambda^{k}(\bar{E})\right), 0\right)$ on the fourth line. This is the content of $[6$, Lemma 7.3 .3 , p. 235, II]. This concludes the proof.

The next corollary follows from the previous one and the discussion at the end of subsection 2.1 .

Corollary 4.5 The Adams operations $\psi^{k}(k \geq 1)$ are ring endomorphisms of $\widehat{K}_{0}(X)$ and satisfy the equation $\psi^{k} \circ \psi^{l}=\psi^{k l}(k, l \geq 1)$.

The fact that the Adams operations are ring endomorphisms is essential to the proof of the arithmetic Adams-Riemann-Roch theorem stated in [17] and proved in [18]. The theorem 4.1 also plays a key role in the proof of the main result of [12] (which is announced in [11]), where it is used to prove the invertibility of the Todd element in a Lefschetz fixed point formula. 


\section{References}

[1] Atiyah M. Tall D.O. Group representations, $\lambda$-rings and the $j$ homomorphism. Topology, 8:253-297, 1969.

[2] Bismut J.-M. Gillet H., Soulé C. Analytic torsion and holomorphic determinant bundles i, ii, iii. Comm. Math. Physics, 115:49-78, 79-126, 301-351, 1988.

[3] Demazure M. Grothendieck A. Schémas en groupes. Springer Lecture Notes 64, 1970.

[4] Fulton W. Harris J. Representation theory. Springer, 1991.

[5] Gasbarri, C. Hermitian vector bundles of rank two and adjoint systems on arithmetic surfaces. Topology, 38: 1161-1174, no. 6 (1999).

[6] Gillet H. Soulé C. Characteristic classes for algebraic vector bundles with hermitian metrics i, ii. Annals of Mathematics, 131:163-203, 205-238, 1990.

[7] Grayson D. Modules projectifs munis d'une métrique sur les anneaux d'entiers de corps de nombres. Comptes Rendus de l'Académie des Sciences, 309:573-575, 1989.

[8] Griffiths P. Harris J. Principles of algebraic geometry. John Wiley, 1978.

[9] Grothendieck A. Berthelot P., Illusie L. Théorie des intersections et théorème de Riemann-Roch.Springer Lecture Notes 225, 1971.

[10] Husemoller D. Fibre bundles. Springer, 1994.

[11] Köhler, K., Roessler, D.: Un théorème du point fixe de Lefschetz en géométrie d'Arakelov. C. R. Acad. Sci. Paris 326, Série I, 719-722, 1998.

[12] Köhler, K., Roessler, D.: A fixed point formula of Lefschetz type in Arakelov geometry I: statement and proof. IHES Preprint M/98/76 (Nov. 1998).

[13] Kratzer C.: $\lambda$-structure en K-théorie algébrique. Comm. Math. Helv., $55: 233-254,1970$.

[14] Lang S. Algebra. Addison-Wesley, 1984.

[15] Meissner A. Arithmetische K-theorie. Diss. Univ. Regensburg, 1993.

[16] Roessler D. Lambda structure en $K$-théorie des fibrés algébriques hermitiens. C. R. Acad. Sci. Paris, 322:251-254, 1996.

[17] Roessler D.Un théorème d'Adams-Riemann-Roch en géométrie d'Arakelov. C. R. Acad. Sci. Paris, 322:749-752, 1996. 
[18] Roessler D. An Adams-Riemann-Roch theorem in Arakelov geometry. To appear in Duke Math. J. 96, 1999.

[19] Serre J.-P. Groupes de Grothendieck des schémas en groupes réductifs déployés. Publications mathématiques de l'IHES, 34:37-52, 1968.

[20] Wang X.Higher order characteristic classes in arithmetic geometry. Harvard Ph. D. thesis, 1992. 